\title{
HOCHSCHILD (CO)HOMOLOGY OF HOPF CROSSED PRODUCTS
}

\author{
Jorge A. Guccione And Juan J. Guccione
}

\begin{abstract}
For a general crossed product $E=A \#_{f} H$, of an algebra $A$ by a Hopf algebra $H$, we obtain complexes smaller than the canonical ones, giving the Hochschild homology and cohomology of $E$. These complexes are equipped with natural filtrations. The spectral sequences associated to them coincide with the ones obtained using a natural generalization of the of the direct method introduced in [H-S]. We also get that if the 2-cocycle $f$ takes its values in a separable subalgebra of $A$, then the Hochschild (co)homology of $E$ with coefficients in $M$ is the (co)homology of $H$ with coefficients in a (co)chain complex.
\end{abstract}

\section{INTRODUCTION}

Let $G$ be a group, $S=\bigoplus S_{g}$ a strongly $G$-graded algebra and $V$ an $S$-bimodule. In $[\mathrm{L}]$ was shown that there is a convergent spectral sequence

$$
E_{r s}^{2}=\mathrm{H}_{r}\left(G, \mathrm{H}_{s}\left(S_{e}, V\right)\right) \Rightarrow \mathrm{H}_{r+s}(S, V),
$$

where $e$ denotes the identity of $G$. In $[S]$ was shown that this result remains valid for $H$-Galois extensions (in his paper the author deals with both the homology and the cohomology of these algebras). An important particular type of $H$-Galois extensions are the crossed products with convolution invertible cocycle $E=A \#{ }_{f} H$, of an algebra $A$ by a Hopf algebra $H$ (for the definition see Section 1). The purpose of our paper is to construct complexes smaller than the canonical ones, given the Hochschild (co)homology of $E$ with coefficients in an arbitrary $E$-bimodule. These complexes are equipped with canonical filtrations. We show that the spectral sequences associated to them coincide with the ones obtained using a natural generalization of the direct method introduced in [H-S], and with the ones constructed in [S] (when these are specialize to crossed products). In the case of group extensions these results were proved in $[\mathrm{E}]$ and $[\mathrm{B}]$.

This paper is organized as follows: in Section 1 a resolution $\left(X_{*}, d_{*}\right)$ of a crossed product $E=A \#_{f} H$ is given. To accomplish this construction we do not use the fact that the cocycle is convolution invertible. Moreover, we give a recursive construction of morphisms $\phi_{*}:\left(X_{*}, d_{*}\right) \rightarrow\left(\mathrm{B}_{*}(E), b_{*}^{\prime}\right)$ and $\psi_{*}:\left(\mathrm{B}_{*}(E), b_{*}^{\prime}\right) \rightarrow\left(X_{*}, d_{*}\right)$, where $\left(\mathrm{B}_{*}(E), b_{*}^{\prime}\right)$ is the normalized Hochschild resolution of $E$, such that $\psi_{*} \phi_{*}=i d$ and we show that $\phi_{*} \psi_{*}$ is homotopically equivalent to the identity map. Consequently

2000 Mathematics Subject Classification. Primary 16E40; Secondary 16W30.

Key words and phrases. Hopf algebra, Hochschild homology.

Supported by UBACYT 01/TW79 and CONICET

We thanks the referee for a substantial improvement in the presentation of Sections 1 and 2 . 
our resolution is a direct sum of the normalized Hochschild resolution. We also recursively construct a homotopy $\phi_{*} \psi_{*} \stackrel{\omega_{*+1}}{\longrightarrow} i d_{*}$. Both the canonical normalized resolution and $\left(X_{*}, d_{*}\right)$ are equipped with natural filtrations, which are preserved by the maps $\phi_{*}, \psi_{*}$ and $\omega_{*+1}$.

In Section 2 , for an $E$-bimodule $M$, we get complexes $\left(\widehat{X}_{*}, \widehat{d}_{*}\right)$ and $\left(\widehat{X}^{*}, \widehat{d}^{*}\right)$, giving the Hochschild homology and cohomology of $E$ with coefficients in $M$, respectively. The filtration of $\left(X_{*}, d_{*}\right)$ induces filtrations on $\left(\widehat{X}_{*}, \widehat{d}_{*}\right)$ and $\left(\widehat{X}^{*}, \widehat{d}^{*}\right)$. So, we obtain converging spectral sequences $E_{r s}^{1}=\mathrm{H}_{r}\left(A, M \otimes \bar{H}^{\otimes^{s}}\right) \Rightarrow \mathrm{H}_{r+s}(E, M)$ and $E_{1}^{r s}=\mathrm{H}^{r}\left(A, \operatorname{Hom}_{k}\left(\bar{H}^{\otimes^{s}}, M\right)\right) \Rightarrow \mathrm{H}^{r+s}(E, M)$. Using the results of Section 1, we get that these spectral sequences are the ones associated to suitable filtrations of the Hochschild normalized chain and cochain complexes $\left(M \otimes \bar{E}^{\otimes^{*}}, b_{*}\right)$ and $\left(\operatorname{Hom}_{k}\left(\bar{E}^{\otimes^{*}}, M\right), b^{*}\right)$, respectively. This allows us to give very simple proofs of the main results of $[\mathrm{H}-\mathrm{S}]$ and $[\mathrm{G}]$.

In Section 3, we show that, if the cocycle is convolution invertible, then the complexes $\left(\widehat{X}_{*}, \widehat{d}_{*}\right)$ and $\left(\widehat{X}^{*}, \widehat{d}^{*}\right)$ are isomorphic to simpler complexes $\left(\bar{X}_{*}, \bar{d}_{*}\right)$ and $\left(\bar{X}^{*}, \bar{d}^{*}\right)$, respectively. Then, we compute the term $E_{r s}^{2}$ and $E_{2}^{r s}$ of the spectral sequences obtained in Section 2. Moreover, using the above mentioned filtrations, we prove that if the 2-cocycle $f$ takes its values in a separable subalgebra of $A$, then the Hochschild (co)homology of $E$ with coefficients in $M$ is the (co)homology of $H$ with coefficients in a (co)chain complex.

In addition to the direct method developed in [H-S], there are another two classical methods to obtain spectral sequences converging to $\mathrm{H}_{*}(E, M)$ and with $E^{2}$ term $\mathrm{H}_{*}\left(H, \mathrm{H}_{*}(A, M)\right)$. Namely the Cartan-Leray and the Grothendieck spectral sequences of a crossed product. In Section 4, we recall these constructions and we prove that these spectral sequences are isomorphic to the one obtained in Section 2. This generalizes the main results of [B]. Similar results are valid in the cohomological setting.

In a first appendix we give a method to construct (under suitable hypothesis) a projective resolution of the $k$-algebra $E$ as $E^{e}=E \otimes E^{\mathrm{op}}$-bimodule, smaller than the canonical one of Hochschild. This method, which can be considered as a variant of the perturbation lemma, is used to prove the main result of Section 1. The boundary maps of the resolution $\left(X_{*}, d_{*}\right)$ are recursively defined in Section 1. In a second appendix we give closed formulas for these maps.

Remark. Let $A$ be an algebra and let $H$ be a $k$-module provided of a base point $1_{H} \in$ $H$. The results of Section 1 and 2 are valid for algebras $A \# H$, whose underlying $k$-module is $A \otimes H$ and whose multiplication map verifies $\left(a \otimes 1_{H}\right)(b \otimes h)=(a b \otimes h)$. We only must modify in an evident way the formulas in Theorem 1.1.3. These type of algebras were introduced in $[\mathrm{Br}]$.

Throughout this paper $k$ denotes an arbitrary commutative base ring, all the algebras are over $k$, the unadorned tensor product is the tensor product over $k$ and $\Upsilon$ denotes the family of all epimorphisms of $E$-bimodules which split as left $E$-module maps.

\section{A RESOlUtion FOR A CROSSED PRODUCT}

Let $A$ be an algebra and $H$ a Hopf algebra. We will use the Sweedler notation $\Delta(h)=h^{(1)} \otimes h^{(2)}$, with the summation understood and superindices instead of 
subindices. Recall some definitions of [B-C-M] and [D-T]. A weak action of $H$ on $A$ is a bilinear map $(h, a) \mapsto a^{h}$ from $H \times A$ to $A$ such that, for $h \in H, a, b \in A$

1) $(a b)^{h}=a^{h^{(1)}} b^{h^{(2)}}$

2) $1^{h}=\epsilon(h) 1$,

3) $a^{1}=a$.

Let $A$ be an algebra and $H$ a Hopf algebra with a weak action on $A$. Given a $k$-linear map $f: H \otimes H \rightarrow A$, let $A \#_{f} H$ be the algebra (in general non-associative and without 1 ) with underlying $k$-module $A \otimes H$ and multiplication map

$$
(a \otimes h)(b \otimes l)=a b^{h^{(1)}} f\left(h^{(2)}, l^{(1)}\right) \otimes h^{(3)} l^{(2)},
$$

for all $a, b \in A, h, l \in H$. The element $a \otimes h$ of $A \#_{f} H$ will usually be written $a \# h$ to remind us $H$ is weakly acting on $A$. The algebra $A \#_{f} H$ is called a crossed product if it is associative with $1 \# 1$ as identity element. It is easy to check that this happens if and only if $f$ and the weak action satisfy the following conditions:

i) (Normality of $f$ ) for all $h \in H$, we have $f(h, 1)=f(1, h)=\epsilon(h) 1_{A}$,

ii) (Cocycle condition) for all $h, l, m \in H$, we have

$$
f\left(l^{(1)}, m^{(1)}\right)^{h^{(1)}} f\left(h^{(2)}, l^{(2)} m^{(2)}\right)=f\left(h^{(1)}, l^{(1)}\right) f\left(h^{(2)} l^{(2)}, m\right),
$$

iii) (Twisted module condition) for all $h, l \in H, a \in A$ we have

$$
\left(a^{l^{(1)}}\right)^{h^{(1)}} f\left(h^{(2)}, l^{(2)}\right)=f\left(h^{(1)}, l^{(1)}\right) a^{h^{(2)} l^{(2)}} .
$$

In this section we obtain a resolution $\left(X_{*}, d_{*}\right)$ of a crossed product $E=A \#{ }_{f} H$ as an $E$-bimodule, which is simpler than the canonical one of Hochschild. To begin, we fix some notations:

1) For each algebra $B$, we put $\bar{B}=B / k$. Moreover, given $b \in B$ we also let $b$ denote the class of $b$ in $\bar{B}$.

2) For each $k$-module $V$ we write $V^{\otimes^{l}}=V \otimes \cdots \otimes V$ ( $l$ times), and for each algebra $B$ we write $\mathrm{B}_{l}(B)=B \otimes \bar{B}^{\otimes^{l}} \otimes B$.

3) Given $a_{0} \otimes \cdots \otimes a_{r} \in A^{\otimes^{r+1}}$ and $0 \leq i<j \leq r$, we write $\mathbf{a}_{i j}=a_{i} \otimes \cdots \otimes a_{j}$.

4) Given $h_{0} \otimes \cdots \otimes h_{s} \in H^{\otimes^{s+1}}$ and $0 \leq i<j \leq s$, we write $\mathbf{h}_{i j}=h_{i} \otimes \cdots \otimes h_{j}$.

\subsection{The resolution $\left(X_{*}, d_{*}\right)$}

Let $Y_{s}=E \otimes_{A}(E / A)^{\left(\otimes_{A}\right)^{s}} \otimes_{A} E$ and $X_{r s}=E \otimes_{A}(E / A)^{\left(\otimes_{A}\right)^{s}} \otimes \bar{A}^{\otimes^{r}} \otimes E$, for all $r, s \geq 0$. Consider the diagram of $E$-bimodules and $E$-bimodule maps

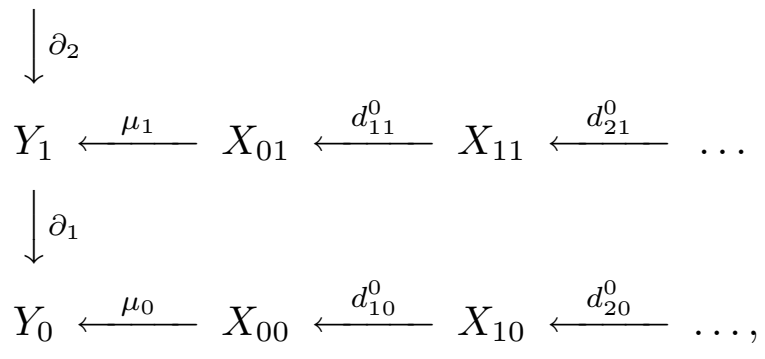


where $\left(Y_{*}, \partial_{*}\right)$ is the normalized Hochschild resolution of the algebra inclusion $A \subseteq$ $E$, in the relative sense, introduced in [G-S]; for each $s \geq 0$, the complex $\left(X_{* s}, d_{* s}^{0}\right)$ is the normalized bar resolution of $A$, tensored on the left over $A$ with $E \otimes_{A}$ $(E / A)^{\left(\otimes_{A}\right)^{s}}$, and on the right over $A$ with $E$; and for each $s \geq 0$, the map $\mu_{s}$ is the canonical projection.

Note that $X_{r s} \simeq E \otimes \bar{H}^{\otimes^{s}} \otimes \bar{A}^{\otimes^{r}} \otimes E$. Moreover, each one of the rows of this diagram is contractible as a left $E$-module complex. A contracting homotopy $\sigma_{0 s}^{0}: Y_{s} \rightarrow X_{0 s}$ and $\sigma_{r+1, s}^{0}: X_{r s} \rightarrow X_{r+1, s}$ of the $s$-th row, is given by

$$
\begin{aligned}
& \sigma_{0 s}^{0}\left(\mathbf{x} \otimes_{A} a \# h\right)=\mathbf{x}_{0, s-1} \otimes_{A} x_{s} a \otimes 1 \# h, \\
& \sigma_{r+1, s}^{0}(\mathbf{x} \otimes \mathbf{a} \otimes a \# h)=(-1)^{r+1} \mathbf{x} \otimes \mathbf{a} \otimes a \otimes 1 \# h,
\end{aligned}
$$

where $\mathbf{x}=x_{0} \otimes_{A} \cdots \otimes_{A} x_{s} \in E \otimes_{A}(E / A)^{\left(\otimes_{A}\right)^{s}}$ and $\mathbf{a}=a_{1} \otimes \cdots \otimes a_{r} \in \bar{A}^{\otimes^{r}}$. So, we are in the situation considered in Appendix A. We define $E$-bimodule maps $d_{r s}^{l}: X_{r s} \rightarrow X_{r+l-1, s-l}(r \geq 0$ and $1 \leq l \leq s)$ recursively, by:

$$
d_{r s}^{l}(\mathbf{x})= \begin{cases}-\sigma_{0, s-1}^{0} \partial_{s} \mu_{s}(\mathbf{x}) & \text { if } r=0 \text { and } l=1, \\ -\sum_{j=1}^{l-1} \sigma_{l-1, s-l}^{0} d_{j-1, s-j}^{l-j} d_{0 s}^{j}(\mathbf{x}) & \text { if } r=0 \text { and } 1<l \leq s, \\ -\sum_{j=0}^{l-1} \sigma_{r+l-1, s-l}^{0} d_{r+j-1, s-j}^{l-j} d_{r s}^{j}(\mathbf{x}) & \text { if } r>0\end{cases}
$$

for $\mathbf{x} \in A \otimes_{A}(E / A)^{\left(\otimes_{A}\right)^{s}} \otimes \bar{A}^{\otimes^{r}} \otimes k$.

Theorem 1.1.1. Let $\mu: X_{00} \rightarrow E$ be the multiplication map. There is a $\Upsilon$ projective resolution of $E$

$$
E \stackrel{-\mu}{\longleftarrow} X_{0} \stackrel{d_{1}}{\longleftarrow} X_{1} \stackrel{d_{2}}{\longleftarrow} X_{2} \stackrel{d_{3}}{\longleftarrow} X_{3} \stackrel{d_{4}}{\longleftarrow} X_{4} \stackrel{d_{5}}{\longleftarrow} X_{5} \stackrel{d_{6}}{\longleftarrow} \ldots
$$

where $X_{n}=\bigoplus_{r+s=n} X_{r s}$ and $d_{n}=\sum_{l=1}^{n} d_{0 n}^{l}+\sum_{r=1}^{n} \sum_{l=0}^{n-r} d_{r, n-r}^{l}$.

Proof. Let $\widetilde{\mu}: Y_{0} \rightarrow E$ be multiplication map. The complex of $E$-bimodules

$$
E \stackrel{\widetilde{\mu}}{\longleftarrow} Y_{0} \stackrel{\partial_{1}}{\longleftarrow} Y_{1} \stackrel{\partial_{2}}{\longleftarrow} Y_{2} \stackrel{\partial_{3}}{\longleftarrow} Y_{3} \stackrel{\partial_{4}}{\longleftarrow} Y_{4} \stackrel{\partial_{5}}{\longleftarrow} Y_{5} \stackrel{\partial_{6}}{\longleftarrow} Y_{6} \stackrel{\partial_{7}}{\longleftarrow} \ldots
$$

is contractible as a complex of left $E$-modules. A chain contracting homotopy $\sigma_{0}^{-1}: E \rightarrow Y_{0}$ and $\sigma_{s+1}^{-1}: Y_{s} \rightarrow Y_{s+1}$, is given by $\sigma_{s+1}^{-1}(\mathbf{x})=(-1)^{s} \mathbf{x} \otimes 1_{E}$. Hence, the theorem follows from Corollary A.2 of Appendix A.

Remark 1.1.2. Let $\sigma_{l, s-l}^{l}: Y_{s} \rightarrow X_{l, s-l}$ and $\sigma_{r+l+1, s-l}^{l}: X_{r s} \rightarrow X_{r+l+1, s-l}$ be the maps recursively defined by

$$
\sigma_{r+l+1, s-l}^{l}=-\sum_{i=0}^{l-1} \sigma_{r+l+1, s-l}^{0} d_{r+i+1, s-i}^{l-i} \sigma_{r+i+1, s-i}^{i} \quad(0<l \leq s \text { and } r \geq-1) .
$$

We will prove, in Corollary A.2, that the family $\bar{\sigma}_{0}: E \rightarrow X_{0}, \bar{\sigma}_{n+1}: X_{n} \rightarrow X_{n+1}$, defined by $\bar{\sigma}_{0}=\sigma_{00}^{0} \sigma_{0}^{-1}$ and

$$
\bar{\sigma}_{n+1}=-\sum_{l=0}^{n+1} \sigma_{l, n-l+1}^{l} \sigma_{n+1}^{-1} \mu_{n}+\sum_{r=0}^{n} \sum_{l=0}^{n-r} \sigma_{r+l+1, n-r-l}^{l} \quad(n \geq 0),
$$

is a contracting homotopy of the resolution (1) introduced in Theorem 1.1.1. 
Theorem 1.1.3. The following assertions hold:

1) Let $\mathbf{x}=x_{0} \otimes_{A} \cdots \otimes_{A} x_{s} \in E \otimes_{A}(E / A)^{\left(\otimes_{A}\right)^{s}}$ with $x_{s}=a_{s} \# h_{s}$, and $\mathbf{b} \in \bar{A}^{\otimes^{r}}$. We have

$$
\begin{aligned}
d_{r s}^{1}\left(\mathbf{x} \otimes \mathbf{b} \otimes 1_{E}\right) & =\sum_{i=0}^{s-1}(-1)^{i+r} \mathbf{x}_{0, i-1} \otimes_{A} x_{i} x_{i+1} \otimes_{A} \mathbf{x}_{i+2, s} \otimes \mathbf{b} \otimes 1_{E} \\
& +(-1)^{r+s} \mathbf{x}_{0, s-2} \otimes_{A} x_{s-1} a_{s} \otimes \mathbf{b}^{h_{s}^{(1)}} \otimes 1 \# h_{s}^{(2)} .
\end{aligned}
$$

2) For each $l \geq 2$, the image of $d_{r s}^{l}$ is contained in the $k$-submodule of $X_{r+l-1, s-l}$ generated by all the elementary tensors $x_{0} \otimes_{A} \cdots \otimes_{A} x_{s-l} \otimes a_{1} \otimes \cdots \otimes a_{r+l-1}$ with $l-1$ of the $a_{i}$ 's in the image of the cocycle $f$.

Proof. The computation of $d_{r s}^{1}$ can be obtained easily by induction on $r$, using that $d_{0 s}^{1}=-\sigma_{0, s-1}^{0} \partial_{s} \mu_{s}^{0}$ and $d_{r s}^{1}=-\sigma_{r, s-1}^{0} d_{r-1, s}^{1} d_{r s}^{0}$, for $r \geq 1$. The assertion for $d_{r s}^{l}$, with $l \geq 2$, follows by induction on $l$ and $r$, using the recursive definition of $d_{r s}^{l}$.

In Appendix B we will give precise formulas for the maps $d_{r s}^{l}$ 's.

\subsection{Comparison with the canonical resolution}

Let $\left(\mathrm{B}_{*}(E), b_{*}^{\prime}\right)$ be the normalized Hochschild resolution of $E$. As is well known, the complex

$$
E \stackrel{\mu}{\longleftarrow} \mathrm{B}_{0}(E) \stackrel{b_{1}^{\prime}}{\longleftarrow} \mathrm{B}_{1}(E) \stackrel{b_{2}^{\prime}}{\longleftarrow} \mathrm{B}_{2}(E) \stackrel{b_{3}^{\prime}}{\longleftarrow} \mathrm{B}_{3}(E) \stackrel{b_{4}^{\prime}}{\longleftarrow} \ldots
$$

is contractible as a complex of left $E$-modules, with contracting homotopy $\xi_{n}(\mathbf{x})=$ $(-1)^{n} \mathbf{x} \otimes 1$. Let $\bar{\sigma}_{*}$ be the contracting homotopy of (1) introduced in Remark 1.1.2. Let $\phi_{*}:\left(X_{*}, d_{*}\right) \rightarrow\left(\mathrm{B}_{*}(E), b_{*}^{\prime}\right)$ and $\psi_{*}:\left(\mathrm{B}_{*}(E), b_{*}^{\prime}\right) \rightarrow\left(X_{*}, d_{*}\right)$ be the morphisms of $E$-bimodule complexes, recursively defined by $\phi_{0}=i d, \psi_{0}=i d, \phi_{n+1}(\mathbf{x} \otimes 1)=$ $\xi_{n+1} \phi_{n} d_{n+1}(\mathbf{x} \otimes 1)$ and $\psi_{n+1}(\mathbf{y} \otimes 1)=\bar{\sigma}_{n+1} \psi_{n} b_{n+1}^{\prime}(\mathbf{y} \otimes 1)$.

Proposition 1.2.1. $\psi_{*} \phi_{*}=i d_{*}$ and $\phi_{*} \psi_{*}$ is homotopically equivalent to the identity map. A homotopy $\phi_{*} \psi_{*} \stackrel{\omega_{*+1}}{\longrightarrow} i d_{*}$ is recursively defined by $\omega_{1}=0$ and $\omega_{n+1}(\mathbf{x})=\xi_{n+1}\left(\phi_{n} \psi_{n}-i d-\omega_{n} b_{n}^{\prime}\right)(\mathbf{x})$, for $\mathbf{x} \in E \otimes \bar{E}^{\otimes^{n}} \otimes k$.

Proof. We prove both assertions by induction. Let $U_{n}=\phi_{n} \psi_{n}-i d_{n}$ and $T_{n}=$ $U_{n}-\omega_{n} b_{n}^{\prime}$. Assuming that $b_{n}^{\prime} \omega_{n}+\omega_{n-1} b_{n-1}^{\prime}=U_{n-1}$, we get that, on $E \otimes \bar{E}^{\otimes^{n}} \otimes k$,

$$
\begin{aligned}
b_{n+1}^{\prime} \omega_{n+1}+\omega_{n} b_{n}^{\prime} & =b_{n+1}^{\prime} \xi_{n+1} T_{n}+\omega_{n} b_{n}^{\prime} \\
& =T_{n}-\xi_{n} b_{n}^{\prime} T_{n}+\omega_{n} b_{n}^{\prime} \\
& =U_{n}-\xi_{n} U_{n-1} b_{n}^{\prime}+\xi_{n} b_{n}^{\prime} \omega_{n} b_{n}^{\prime} \\
& =U_{n}-\xi_{n} U_{n-1} b_{n}^{\prime}+\xi_{n} T_{n-1} b_{n}^{\prime}=U_{n} .
\end{aligned}
$$

Hence, $b_{n+1}^{\prime} \omega_{n+1}+\omega_{n} b_{n}^{\prime}=U_{n}$ on $\mathrm{B}_{n}(E)$. Next, we prove that $\psi_{*} \phi_{*}=i d_{*}$. It is clear that $\psi_{0} \phi_{0}=i d_{0}$. Assume that $\psi_{n} \phi_{n}=i d_{n}$. Since

$$
\phi_{n+1}\left(E \otimes_{A}(E / A)^{\left(\otimes_{A}\right)^{s}} \otimes \bar{A}^{\otimes^{n+1-s}} \otimes k\right) \subseteq E \otimes \bar{E}^{\otimes^{n+1}} \otimes k
$$


we have that, on $A \otimes_{A}(E / A)^{\left(\otimes_{A}\right)^{s}} \otimes \bar{A}^{\otimes^{n+1-s}} \otimes k$,

$$
\begin{aligned}
\psi_{n+1} \phi_{n+1} & =\bar{\sigma}_{n+1} \psi_{n} b_{n+1}^{\prime} \phi_{n+1} \\
& =\bar{\sigma}_{n+1} \psi_{n} b_{n+1}^{\prime} \xi_{n+1} \phi_{n} d_{n+1} \\
& =\bar{\sigma}_{n+1} \psi_{n} \phi_{n} d_{n+1}-\bar{\sigma}_{n+1} \psi_{n} \xi_{n} b_{n}^{\prime} \phi_{n} d_{n+1} \\
& =\bar{\sigma}_{n+1} d_{n+1}=i d_{n+2}-d_{n+2} \bar{\sigma}_{n+2} .
\end{aligned}
$$

So, to end the proof it suffices to see that $\bar{\sigma}_{n+2}\left(A \otimes_{A}(E / A)^{\left(\otimes_{A}\right)^{s}} \otimes \bar{A}^{\otimes^{n+1-s}} \otimes k\right)=0$, which follows easily from the definition of $\bar{\sigma}_{*}$.

Let $F^{i}\left(X_{n}\right)=\bigoplus_{0 \leq s \leq i} E \otimes_{A}(E / A)^{\left(\otimes_{A}\right)^{s}} \otimes \bar{A}^{\otimes^{n-s}} \otimes k$ and let $F^{i}\left(\mathrm{~B}_{n}(E)\right)$ be the subbimodule of $\mathrm{B}_{n}(E)$ generated by the tensors $1 \otimes x_{1} \otimes \cdots \otimes x_{n} \otimes 1$ such that at least $n-i$ of the $x_{j}$ 's belong to $A$. The normalized Hochschild resolution $\left(\mathrm{B}_{*}(E), b_{*}^{\prime}\right)$ and the resolution $\left(X_{*}, d_{*}\right)$ are filtered by $F^{0}\left(\mathrm{~B}_{*}(E)\right) \subseteq F^{1}\left(\mathrm{~B}_{*}(E)\right) \subseteq F^{2}\left(\mathrm{~B}_{*}(E)\right) \subseteq \ldots$ and $F^{0}\left(X_{*}\right) \subseteq F^{1}\left(X_{*}\right) \subseteq F^{2}\left(X_{*}\right) \subseteq \ldots$, respectively

Proposition 1.2.2. The maps $\phi_{*}, \psi_{*}$ and $\omega_{*+1}$ preserve filtrations.

Proof. Let $Q_{j}^{i}=E \otimes_{A}(E / A)^{\left(\otimes_{A}\right)^{i}} \otimes \bar{A}^{\otimes^{n-j}} \otimes k$. We claim that

a) $\bar{\sigma}_{n+1}\left(F^{i}\left(X_{n}\right)\right) \subseteq F^{i}\left(X_{n+1}\right)$ for all $0 \leq i<n$,

b) $\bar{\sigma}_{n+1}\left(E \otimes_{A}(E / A)^{\left(\otimes_{A}\right)^{i}} \otimes \bar{A}^{\otimes^{n-i}} \otimes A\right) \subseteq Q_{i-1}^{i}+F^{i-1}\left(X_{n+1}\right)$ for all $0 \leq i \leq n$,

c) $\bar{\sigma}_{n+1}\left(E \otimes_{A}(E / A)^{\left(\otimes_{A}\right)^{n}} \otimes E\right) \subseteq E \otimes_{A}(E / A)^{\left(\otimes_{A}\right)^{n+1}} \otimes k+F^{n}\left(X_{n+1}\right)$,

d) $\psi_{n}\left(F^{i}\left(\mathrm{~B}_{n}(E)\right) \cap E \otimes \bar{E}^{\otimes^{n}} \otimes k\right) \subseteq Q_{i}^{i}+F^{i-1}\left(X_{n}\right)$ for all $0 \leq i \leq n$.

In fact a), b) and c) follow immediately from the definition of $\bar{\sigma}_{n+1}$. Suppose d) is valid for $n$. Let $\mathbf{x}=x_{0} \otimes \cdots \otimes x_{n+1} \otimes 1 \in F^{i}\left(\mathrm{~B}_{n+1}(E)\right) \cap E \otimes \bar{E}^{\otimes^{n+1}} \otimes k$, with $0 \leq i \leq n+1$. Using a) and b), we get that for $1 \leq j \leq n$,

$$
\begin{aligned}
\bar{\sigma}_{n+1}\left(\psi_{n}\left(\mathbf{x}_{0, j-1} \otimes x_{j} x_{j+1} \otimes \mathbf{x}_{j+2, n+1} \otimes 1\right)\right) & \subseteq \bar{\sigma}_{n+1}\left(Q_{i}^{i}+F^{i-1}\left(X_{n}\right)\right) \\
& \subseteq Q_{i-1}^{i}+F^{i-1}\left(X_{n+1}\right) .
\end{aligned}
$$

Since $\psi_{n+1}(\mathbf{x})=\bar{\sigma}_{n+1} \psi_{n} b_{n+1}^{\prime}(\mathbf{x})$, to prove d) for $n+1$ we only must check that $\bar{\sigma}_{n+1}\left(\psi_{n}\left(\mathbf{x}_{0, n+1}\right)\right) \subseteq Q_{i-1}^{i}+F^{i-1}\left(X_{n+1}\right)$. If $x_{n+1} \in A$, then using a), b) and the inductive hypothesis, we get

$$
\begin{aligned}
& \bar{\sigma}_{n+1}\left(\psi_{n}\left(\mathbf{x}_{0, n+1}\right)\right)=\bar{\sigma}_{n+1}\left(\psi_{n}\left(\mathbf{x}_{0 n} \otimes 1_{E}\right) x_{n+1}\right) \\
& \subseteq \bar{\sigma}_{n+1}\left(E \otimes_{A}(E / A)^{\left(\otimes_{A}\right)^{i}} \otimes \bar{A}^{\otimes^{n-i}} \otimes A+F^{i-1}\left(X_{n}\right)\right) \\
& \subseteq Q_{i-1}^{i}+F^{i-1}\left(X_{n+1}\right) \text {, }
\end{aligned}
$$

and if $x_{n+1} \notin A$, then $\mathbf{x}_{0, n+1} \in F^{i-1}\left(\mathrm{~B}_{n}(E)\right)$, which together with a), c) and the inductive hypothesis, implies that

$$
\bar{\sigma}_{n+1}\left(\psi_{n}\left(\mathbf{x}_{0, n+1}\right)\right) \subseteq \bar{\sigma}_{n+1}\left(F^{i-1}\left(X_{n}\right)\right) \subseteq Q_{i-1}^{i}+F^{i-1}\left(X_{n+1}\right) .
$$

From d) it follows immediately that $\psi_{*}$ preserves filtrations. Next, assuming that $\phi_{n}$ preserves filtrations, we prove that $\phi_{n+1}$ does. Let $\mathbf{y}=\mathbf{x} \otimes \mathbf{a} \otimes 1$, where 
$\mathbf{x}=x_{0} \otimes_{A} \cdots \otimes_{A} x_{i} \in E \otimes_{A}(E / A)^{\left(\otimes_{A}\right)^{i}}$ and $\mathbf{a} \otimes 1=a_{1} \otimes \cdots \otimes a_{n+1-i} \otimes 1 \in \bar{A}^{\otimes^{n+1-i}} \otimes k$. Since $\phi_{n+1}(\mathbf{y})=\xi_{n+1} \phi_{n} d_{n}(\mathbf{y})$ and

$$
\begin{aligned}
\xi_{n+1}\left(\phi_{n}\left(d_{r s}^{l}(\mathbf{y})\right)\right) & \subseteq \xi_{n+1}\left(\phi_{n}\left(F^{i-l}\left(X_{n}\right)\right)\right) \\
& \subseteq \xi_{n+1}\left(F^{i-l}\left(\mathrm{~B}_{n}(E)\right)\right) \subseteq F^{i-l+1}\left(\mathrm{~B}_{n+1}(E)\right)
\end{aligned}
$$

it suffices to see that $\xi_{n+1}\left(\phi_{n}\left(d_{r s}^{0}(\mathbf{y})\right)\right) \subseteq F^{i}\left(\mathrm{~B}_{n+1}(E)\right)$. But

$$
\begin{aligned}
\xi_{n+1} \phi_{n} d_{r s}^{0}(\mathbf{y}) & =(-1)^{r} \xi_{n+1} \phi_{n}(\mathbf{x} \otimes \mathbf{a}) \\
& =(-1)^{r} \xi_{n+1}\left(\phi_{n}\left(\mathbf{x}_{0 i} \otimes \mathbf{a}_{1, n-i} \otimes 1\right) a_{n+1-i}\right) \\
& \subseteq \xi_{n+1}\left(F^{i}\left(\mathrm{~B}_{n}(E)\right) \cap E \otimes \bar{E}^{\otimes^{n}} \otimes A\right) \\
& \subseteq F^{i}\left(\mathrm{~B}_{n+1}(E)\right),
\end{aligned}
$$

since $\phi_{n}\left(Q_{i}^{i}\right) \subseteq E \otimes \bar{E}^{\otimes^{n}} \otimes k$. Next, we prove that $\omega_{*}$ preserves filtrations. Assume that $\omega_{n}$ does. Let $\mathbf{x}=x_{0} \otimes \cdots \otimes x_{n} \otimes 1 \in F^{i}\left(\mathrm{~B}_{n}(E)\right) \cap E \otimes \bar{E}^{\otimes^{n}} \otimes k$. It is evident that $\omega_{n+1}(\mathbf{x})=\xi_{n+1} \phi_{n} \psi_{n}(\mathbf{x})-\xi_{n+1} \omega_{n} b_{n}^{\prime}(\mathbf{x})$. From d) we get

$$
\xi_{n+1} \phi_{n} \psi_{n}(\mathbf{x}) \in \xi_{n+1} \phi_{n}\left(Q_{i}^{i}+F^{i-1}\left(X_{n}\right)\right) \subseteq \xi_{n+1}\left(F^{i-1}\left(\mathrm{~B}_{n}(E)\right)\right) \subseteq F^{i}\left(\mathrm{~B}_{n}(E)\right),
$$

since $\xi_{n+1}\left(\phi_{n}\left(Q_{i}^{i}\right)\right) \subseteq \xi_{n+1}\left(E \otimes \bar{E}^{\otimes^{n}} \otimes k\right)=0$. To finish, it remains to check that $\xi_{n+1} \omega_{n} b_{n}^{\prime}(\mathbf{x}) \subseteq F^{i}\left(\mathrm{~B}_{n}(E)\right)$. Since $\omega_{n}\left(E \otimes \bar{E}^{\otimes^{n-1}} \otimes k\right) \subseteq E \otimes \bar{E}^{\otimes^{n}} \otimes k$, we have $\xi_{n+1} \omega_{n} b_{n}^{\prime}(\mathbf{x})=(-1)^{n-1} \xi_{n+1} \omega_{n}\left(\mathbf{x}_{0 n}\right)$. Hence, if $x_{n} \in A$, then

$$
\begin{aligned}
\xi_{n+1} \omega_{n} b_{n}^{\prime}(\mathbf{x}) & =(-1)^{n-1} \xi_{n+1}\left(\omega_{n}\left(\mathbf{x}_{0, n-1} \otimes 1\right) x_{n}\right) \\
& \subseteq \xi_{n+1}\left(F^{i}\left(\mathrm{~B}_{n}(E)\right) \cap E \otimes \bar{E}^{\otimes^{n}} \otimes A\right) \\
& \subseteq F^{i}\left(\mathrm{~B}_{n+1}(E)\right),
\end{aligned}
$$

and if $x_{n} \notin A$, then $\mathbf{x}_{0 n} \in F^{i-1}\left(\mathrm{~B}_{n-1}(E)\right)$, and so

$$
\xi_{n+1} \omega_{n} b_{n}^{\prime}(\mathbf{x})=(-1)^{n-1} \xi_{n+1} \omega_{n}\left(\mathbf{x}_{0 n}\right) \subseteq \xi_{n+1}\left(F^{i-1}\left(\mathrm{~B}_{n}(E)\right)\right) \subseteq F^{i}\left(\mathrm{~B}_{n+1} E\right) .
$$

\section{The Hochschild (CO)homology of A CROssed product}

Let $E=A \#_{f} H$ and let $M$ be an E-bimodule. In this section we use Theorem 1.1.1 in order to construct complexes, smaller than the canonical ones, giving the Hochschild homology and cohomology of $A$ with coefficients in $M$, respectively. These complexes have natural filtrations that allow us to obtain spectral sequences converging to $\mathrm{H}_{*}(E, M)$ and $\mathrm{H}^{*}(E, M)$, respectively. We compare these spectral sequences with the ones obtained from a generalization of the Hochschild-Serre direct method.

Theorem 2.1. The Hochschild homology of E with coefficients in $M$ is the homology of $M \otimes_{E^{e}}\left(X_{*}, d_{*}\right)$.

Proof. It is immediate. 
Let $\widehat{X}_{r s}=M \otimes_{A}(E / A)^{\left(\otimes_{A}\right)^{s}} \otimes \bar{A}^{\otimes^{r}}$. It is well known that $\widehat{X}_{r s} \simeq M \otimes_{E^{e}} X_{r s}$. Let $\widehat{d}_{r s}^{l}: \widehat{X}_{r s} \rightarrow \widehat{X}_{r+l-1, s-l}$ be the map induced by $i d_{M} \otimes_{E^{e}} d_{r s}^{l}$. It is easy to see that $\widehat{d}_{r s}^{0}$ is the boundary map of the normalized chain Hochschild complex of $A$ with coefficients in $M \otimes_{A}(E / A)^{\left(\otimes_{A}\right)^{s}}$. With the above identifications the complex $M \otimes_{E^{e}}\left(X_{*}, d_{*}\right)$ becomes $\left(\widehat{X}_{*}, \widehat{d}_{*}\right)$, where

$$
\widehat{X}_{n}=\bigoplus_{r+s=n} \widehat{X}_{r s} \quad \text { and } \quad \widehat{d}_{n}:=\sum_{l=1}^{n} \widehat{d}_{0 n}^{l}+\sum_{r=1}^{n} \sum_{l=0}^{n-r} \widehat{d}_{r, n-r}^{l} .
$$

Let $F^{i}\left(\widehat{X}_{n}\right)=\bigoplus_{0 \leq s \leq i} \widehat{X}_{n-s, s}$. Clearly $F^{0}\left(\widehat{X}_{*}\right) \subseteq F^{1}\left(\widehat{X}_{*}\right) \subseteq \ldots$ is a filtration of $\left(\widehat{X}_{*}, \widehat{d}_{*}\right)$. Using this fact we obtain:

Corollary 2.2. There is a convergent spectral sequence

$$
E_{r s}^{1}=\mathrm{H}_{r}\left(A, M \otimes_{A}(E / A)^{\left(\otimes_{A}\right)^{s}}\right) \Rightarrow \mathrm{H}_{r+s}(E, M) .
$$

The normalized Hochschild complex $\left(M \otimes \bar{E}^{\otimes^{*}}, b_{*}\right)$ is filtered by

$$
F^{0}\left(M \otimes \bar{E}^{\otimes^{*}}\right) \subseteq F^{1}\left(M \otimes \bar{E}^{\otimes^{*}}\right) \subseteq F^{2}\left(M \otimes \bar{E}^{\otimes^{*}}\right) \subseteq \ldots
$$

where $F^{i}\left(M \otimes \bar{E}^{\otimes^{n}}\right)$ is the $k$-submodule of $M \otimes \bar{E}^{\otimes^{n}}$ generated by the tensors $m \otimes x_{1} \otimes \cdots \otimes x_{n}$ such that at least $n-i$ of the $x_{j}$ 's belong to $A$. The spectral sequence associated to this filtration is called the homological Hochschild-Serre spectral sequence. Since, for each extension of groups $N \subseteq G$, with $N$ a normal subgroup, $k[G]$ is a crossed product of $k[G / N]$ on $k[N]$, the following theorem (joint with Corollary 3.1.3 below) gives, as a particular case, the homological version of the main results of $[\mathrm{H}-\mathrm{S}]$.

Theorem 2.3. The homological Hochschild-Serre spectral sequence is isomorphic to the one obtained in Corollary 2.2.

Proof. It is immediate that the filtrations of $\left(\widehat{X}_{*}, \widehat{d}_{*}\right)$ and $\left(M \otimes \bar{E}^{\otimes^{*}}, b_{*}\right)$ are the ones induced by the filtrations of $\left(X_{*}, d_{*}\right)$ and $\left(\mathrm{B}_{*}(E), b_{*}^{\prime}\right)$, introduced above Proposition 1.1.2, respectively. Hence, the result follows from Propositions 1.2.1 and 1.2 .2 .

Theorem 2.4. The Hochschild cohomology of $E$ with coefficients in $M$ is the cohomology of $\operatorname{Hom}_{E^{e}}\left(\left(X_{*}, d_{*}\right), M\right)$.

Proof. It is immediate.

Let $\widehat{X}^{r s}=\operatorname{Hom}_{A}\left((E / A)^{\left(\otimes_{A}\right)^{s}} \otimes \bar{A}^{\otimes^{r}}, M\right) \simeq \operatorname{Hom}_{k}\left(\bar{A}^{\otimes^{r}}, \operatorname{Hom}_{A}\left((E / A)^{\left(\otimes_{A}\right)^{s}}, M\right)\right)$. Clearly $\widehat{X}^{r s} \simeq \operatorname{Hom}_{E^{e}}\left(X_{r s}, M\right)$. Let $\widehat{d}_{l}^{r s}: \widehat{X}^{r+l-1, s-l} \rightarrow \widehat{X}^{r s}$ be the map induced by $\operatorname{Hom}_{E^{e}}\left(d_{r s}^{l}, M\right)$. It is easy to see that $\widehat{d}_{0}^{r s}$ is the boundary map of the normalized cochain Hochschild complex of $A$ with coefficients in $\operatorname{Hom}_{A}\left((E / A)^{\left(\otimes_{A}\right)^{s}}, M\right)$. With the above identifications $\operatorname{Hom}_{E^{e}}\left(\left(X_{*}, d_{*}\right), M\right)$ becomes $\left(\widehat{X}^{*}, \widehat{d}^{*}\right)$, where

$$
\widehat{X}^{n}=\bigoplus_{r+s=n} \widehat{X}^{r s} \quad \text { and } \quad \widehat{d}^{n}:=\sum_{l=1}^{n} \widehat{d}_{l}^{0 n}+\sum_{r=1}^{n} \sum_{l=0}^{n-r} \widehat{d}_{l}^{r, n-r} .
$$

Let $F_{i}\left(\widehat{X}^{n}\right)=\bigoplus_{s \geq i} \widehat{X}^{n-s, s}$. Clearly $F_{0}\left(\widehat{X}^{*}\right) \supseteq F_{1}\left(\widehat{X}^{*}\right) \supseteq F_{2}\left(\widehat{X}^{*}\right) \supseteq \ldots$ is a filtration of $\left(\widehat{X}^{*}, \widehat{d}^{*}\right)$. Using this fact we obtain: 
Corollary 2.5. There is a convergent spectral sequence

$$
E_{1}^{r s}=\mathrm{H}^{r}\left(A, \operatorname{Hom}_{A}\left((E / A)^{\left(\otimes_{A}\right)^{s}}, M\right)\right) \Rightarrow \mathrm{H}^{r+s}(E, M) .
$$

Let $F_{i}\left(\operatorname{Hom}_{k}\left(\bar{E}^{\otimes^{n}}, M\right)\right)$ be the $k$-submodule of $\operatorname{Hom}_{k}\left(\bar{E}^{\otimes^{n}}, M\right)$ consisting of maps $\varphi \in \operatorname{Hom}_{k}\left(\bar{E}^{\otimes^{n}}, M\right)$, for which $\varphi\left(x_{1} \otimes \cdots \otimes x_{n}\right)=0$ whenever $n-i$ of the $x_{j}$ 's belong to $A$. The normalized Hochschild complex $\left(\operatorname{Hom}_{k}\left(\bar{E}^{\otimes^{*}}, M\right), b^{*}\right)$ is filtered by

$$
F_{0}\left(\operatorname{Hom}_{k}\left(\bar{E}^{*}, M\right)\right) \supseteq F_{1}\left(\operatorname{Hom}_{k}\left(\bar{E}^{*}, M\right)\right) \supseteq F_{2}\left(\operatorname{Hom}_{k}\left(\bar{E}^{*}, M\right)\right) \supseteq \ldots
$$

The spectral sequence associated to this filtration is called the cohomological Hochschild-Serre spectral sequence. The following theorem, joint with Corollary 3.2.3 below, gives, as a particular case, the main results of [H-S].

Theorem 2.6. The cohomological Hochschild-Serre spectral sequence is isomorphic to the one obtained in Corollary 2.5.

Proof. It is immediate that the filtrations of $\left(\widehat{X}^{*}, \widehat{d}^{*}\right)$ and $\left(\operatorname{Hom}_{k}\left(\bar{E}^{\otimes^{*}}, M\right), b^{*}\right)$ are the ones induced by the filtrations of $\left(X_{*}, d_{*}\right)$ and $\left(\mathrm{B}_{*}(E), b_{*}^{\prime}\right)$, introduced above Proposition 1.1.2, respectively. Hence, the result follows from Propositions 1.2.1 and 1.2.2.

\section{The Hochschild (CO)homology of A CROSSED PRODUCT WITH INVERTIBLE COCYCLE}

Let $E=A \#{ }_{f} H$ and let $M$ be an $E$-bimodule. Assume that the cocycle $f$ is invertible. Then, the map $h \mapsto 1 \# h$ is convolution invertible and its inverse is the map $h \mapsto(1 \# h)^{-1}=f^{-1}\left(S\left(h^{(2)}\right), h^{(3)}\right) \# S\left(h^{(1)}\right)$. Under this hypothesis, we prove that the complexes $\widehat{X}_{*}(E, M)$ and $\widehat{X}^{*}(E, M)$ of Section 2 are isomorphic to simpler complexes. We use these new complexes in order to compute the term $E^{2}$ of the spectral sequences obtained in Section 2. Moreover, using a theorem of Gerstenhaber and Schack, we prove that if the 2-cocycle $f$ takes its values in a separable subalgebra of $A$, then the Hochschild (co)homology of $E$ with coefficients in $M$ is the (co)homology of $H$ with coefficients in a (co)chain complex.

\subsection{Hochschild homology}

Let $\bar{X}_{r s}=M \otimes \bar{A}^{\otimes^{r}} \otimes \bar{H}^{\otimes^{s}}$. The map $\theta_{r s}: \widehat{X}_{r s} \rightarrow \bar{X}_{r s}$, defined by $\theta_{r s}\left(m \otimes_{A}\left(1 \# h_{1}\right) \otimes_{A} \cdots \otimes_{A}\left(1 \# h_{s}\right) \otimes \mathbf{a}\right)=m\left(1 \# h_{1}^{(1)}\right) \cdots\left(1 \# h_{s}^{(1)}\right) \otimes \mathbf{a} \otimes h_{1}^{(2)} \otimes \cdots \otimes h_{s}^{(2)}$, where $\mathbf{a}=a_{1} \otimes \cdots \otimes a_{r}$, is an isomorphism. The inverse map of $\theta_{r s}$ is the map $m \otimes \mathbf{a} \otimes h_{1} \otimes \cdots \otimes h_{s} \mapsto m\left(1 \# h_{s}^{(1)}\right)^{-1} \cdots\left(1 \# h_{1}^{(1)}\right)^{-1} \otimes_{A}\left(1 \# h_{1}^{(2)}\right) \otimes_{A} \cdots \otimes_{A}\left(1 \# h_{s}^{(2)}\right) \otimes \mathbf{a}$ Let $\bar{d}_{r s}^{l}: \bar{X}_{r s} \rightarrow \bar{X}_{r+l-1, s-l}$ be the map $\bar{d}_{r s}^{l}:=\theta_{r+l-1, s-l} \widehat{d}_{r s}^{l} \theta_{r s}^{-1}$. 
Theorem 3.1.1. The Hochschild homology of $E$ with coefficients in $M$ is the homology of $\left(\bar{X}_{*}, \bar{d}_{*}\right)$, where

$$
\bar{X}_{n}=\bigoplus_{r+s=n} \bar{X}_{r s} \quad \text { and } \quad \bar{d}_{n}:=\sum_{l=1}^{n} \bar{d}_{0 n}^{l}+\sum_{r=1}^{n} \sum_{l=0}^{n-r} \bar{d}_{r, n-r}^{l}
$$

Proof. This is an immediate consequence of Theorem 2.1 and the fact that the map $\theta_{*}:\left(\widehat{X}_{*}, \widehat{d}_{*}\right) \rightarrow\left(\bar{X}_{*}, \bar{d}_{*}\right)$, given by $\theta_{n}=\sum_{r+s=n} \theta_{r s}$, is an isomorphism.

Note that when $f$ takes its values in $k$, then $\left(\bar{X}_{*}, \bar{d}_{*}\right)$ is the total complex of the double complex $\left(M \otimes \bar{A}^{\otimes^{*}} \otimes \bar{H}^{\otimes^{*}}, \bar{d}_{* *}^{0}, \bar{d}_{* *}^{1}\right)$.

It is easy to see that $\bar{d}_{r s}^{0}$ is the the boundary map of the normalized chain Hochschild complex of $A$ with coefficients in $M$, tensored on the right over $k$ with $i d_{\bar{H}^{\otimes^{s}}}$, and

$$
\begin{aligned}
\bar{d}_{r s}^{1}(\mathbf{x}) & =(-1)^{r+s}\left(1 \# h_{s}^{(3)}\right) m\left(1 \# h_{s}^{(1)}\right)^{-1} \otimes \mathbf{a}_{s}^{h_{s}^{(2)}} \otimes \mathbf{h}_{1, s-1} \\
& +\sum_{i=1}^{s-1}(-1)^{r+i} m \otimes \mathbf{a} \otimes \mathbf{h}_{1, i-1} \otimes h_{i} h_{i+1} \otimes \mathbf{h}_{i+2, s}+(-1)^{r} m \epsilon\left(h_{1}\right) \otimes \mathbf{a} \otimes \mathbf{h}_{2 s},
\end{aligned}
$$

where $\mathbf{x}=m \otimes \mathbf{a} \otimes \mathbf{h}$, with $\mathbf{a}=a_{1} \otimes \cdots \otimes a_{r}$ and $\mathbf{h}=h_{1} \otimes \cdots \otimes h_{s}$.

For each $h \in H$, we have the morphism $\vartheta_{*}^{h}:\left(M \otimes \bar{A}^{\otimes^{*}}, b_{*}\right) \rightarrow\left(M \otimes \bar{A}^{\otimes^{*}}, b_{*}\right)$, defined by $\vartheta_{r}^{h}(m \otimes \mathbf{a})=\left(1 \# h_{s}^{(3)}\right) m\left(1 \# h_{s}^{(1)}\right)^{-1} \otimes \mathbf{a}^{h_{s}^{(2)}}$.

Proposition 3.1.2. For each $h, l \in H$, the endomorphisms of $\mathrm{H}_{*}(A, M)$ induced by $\vartheta_{*}^{h} \vartheta_{*}^{l}$ and by $\vartheta_{*}^{h l}$ coincide. Consequently $\mathrm{H}_{*}(A, M)$ is a left $H$-module.

Proof. By a standard argument it is sufficient to prove it for $\mathrm{H}_{0}(A, M)$, and in this case the result is immediate.

Let $F^{0}\left(\bar{X}_{*}\right) \subseteq F^{1}\left(\bar{X}_{*}\right) \subseteq \ldots$ be the filtration of $\left(\bar{X}_{*}, \bar{d}_{*}\right)$ obtained transporting the one of $\left(\widehat{X}_{*}, \widehat{d}_{*}\right)$, given above Corollary 2.2 , through $\theta_{*}$. It is immediate that $F^{i}\left(\bar{X}_{n}\right)=\bigoplus_{0 \leq s \leq i} \bar{X}_{n-s, s}$. Then, we have the following:

Corollary 3.1.3. if $H$ is flat over $k$, then the spectral sequence of Corollary 2.2 satisfies $E_{r s}^{1}=\mathrm{H}_{r}(A, M) \otimes \bar{H}^{\otimes^{s}}$ and $E_{r s}^{2}=\mathrm{H}_{s}\left(H, \mathrm{H}_{r}(A, M)\right)$.

Let $S$ be a separable subalgebra of $A$. Next we prove that if the 2-cocycle $f$ takes its values in $S$, then the Hochschild homology of $E$ with coefficients in $M$ is the homology of $H$ with coefficients in a chain complex. Assume that $f(h, l) \in S$ for all $h, l \in H$. Let $\widetilde{A}=A / S, \widetilde{A}^{\left(\otimes_{S}\right)^{0}}=S$ and $\widetilde{A}^{\left(\otimes_{S}\right)^{r}}=\widetilde{A} \otimes_{S} \cdots \otimes_{S} \widetilde{A}(r$-times $)$ for $r>0$, and let $M \otimes_{S} \widetilde{A}^{\left(\otimes_{S}\right)^{r}} \otimes_{S}=M \otimes_{A^{e}}\left(A \otimes_{S} \widetilde{A}^{\left(\otimes_{S}\right)^{r}} \otimes_{S} A\right)=M \otimes_{S} \widetilde{A}^{\left(\otimes_{S}\right)^{r}} \otimes_{S^{e}} S$ be the cyclic tensor product over $S$ of $M$ and $\widetilde{A}^{\left(\otimes_{S}\right)^{r}}$ (see [G-S] or [Q]). Using the fact that $f$ takes its values in $S$, it is easy to see that $H$ acts on $\left(M \otimes_{S} \widetilde{A}^{\left(\otimes_{S}\right)^{*}} \otimes_{S}, b_{*}\right)$ via

$$
h \cdot\left(m \otimes_{S} \widetilde{\mathbf{a}}\right)=\left(1 \# h^{(3)}\right) m\left(1 \# h^{(1)}\right)^{-1} \otimes_{S} \widetilde{\mathbf{a}}^{h^{(2)}},
$$

where $m \otimes_{S} \widetilde{\mathbf{a}}=m \otimes_{S} a_{1} \otimes_{S} \cdots \otimes_{S} a_{r} \otimes_{S}$ and $\widetilde{\mathbf{a}}^{h^{(2)}}=a_{1}^{h^{(2)}} \otimes_{S} \cdots \otimes_{S} a_{r}^{h^{(r+1)}} \otimes_{S}$. 
Theorem 3.1.4. The Hochschild homology $\mathrm{H}_{*}(E, M)$, of $E$ with coefficients in $M$, is the homology of $H$ with coefficients in $\left(M \otimes_{S} \widetilde{A}^{\left(\otimes_{S}\right)^{*}} \otimes_{S}, b_{*}\right)$.

Proof. Let $\left(\left(M \otimes_{S} \widetilde{A}^{\left(\otimes_{S}\right)^{*}} \otimes_{S}\right) \otimes \bar{H}^{\otimes^{*}}, \widetilde{d}_{* *}^{0}, \widetilde{d}_{* *}^{1}\right)$ be the double complex with horizontal differentials

$$
\begin{aligned}
\widetilde{d}_{r s}^{0}(\mathbf{x}) & =m a_{1} \otimes_{S} \widetilde{\mathbf{a}}_{2 r} \otimes \mathbf{h}+(-1)^{r} a_{r} m \otimes_{S} \widetilde{\mathbf{a}}_{1, r-1} \otimes \mathbf{h} \\
& +\sum_{i=1}^{r-1}(-1)^{i} m \otimes_{S} \widetilde{\mathbf{a}}_{1, i-1} \otimes_{S} a_{i} a_{i+1} \otimes_{S} \widetilde{\mathbf{a}}_{i+2, r} \otimes \mathbf{h},
\end{aligned}
$$

and vertical differentials

$$
\begin{aligned}
\widetilde{d}_{r s}^{1}(\mathbf{x}) & =(-1)^{r} m \otimes_{s} \widetilde{\mathbf{a}} \otimes \mathbf{h}_{2 s}+(-1)^{r+s}\left(1 \# h_{s}^{(3)}\right) m\left(1 \# h_{s}^{(1)}\right)^{-1} \otimes_{S} \widetilde{\mathbf{a}}_{s}^{h_{s}^{(2)}} \otimes \mathbf{h}_{1, s-1} \\
& +\sum_{i=1}^{s-1}(-1)^{r+i} m \otimes_{S} \widetilde{\mathbf{a}} \otimes h_{1}^{(2)} \otimes \cdots \otimes h_{i-1}^{(2)} \otimes h_{i}^{(2)} h_{i+1}^{(2)} \otimes \mathbf{h}_{i+2, s}
\end{aligned}
$$

where $\mathbf{x}=m \otimes \widetilde{\mathbf{a}} \otimes \mathbf{h}$, with $\widetilde{\mathbf{a}}=a_{1} \otimes_{S} \cdots \otimes_{S} a_{r} \otimes_{S}$ and $\mathbf{h}=h_{1} \otimes \cdots \otimes h_{s}$. Let $\left(\bar{X}_{*}^{S}, \bar{d}_{*}^{S}\right)$ be the total complex of $\left(\left(M \otimes_{S} \widetilde{A}^{\left(\otimes_{S}^{*}\right)} \otimes_{S}\right) \otimes \bar{H}^{\otimes^{*}}, \widetilde{d}_{* *}^{0}, \widetilde{d}_{* *}^{1}\right)$. We must prove that $\mathrm{H}_{*}(E, M)$ is the homology of $\left(\bar{X}_{*}^{S}, \bar{d}_{*}^{S}\right)$. Let $\pi_{*}:\left(\bar{X}_{*}, \bar{d}_{*}\right) \rightarrow\left(\bar{X}_{*}^{S}, \bar{d}_{*}^{S}\right)$ be the map $m \otimes \mathbf{a} \otimes \mathbf{h} \mapsto m \otimes_{S} \widetilde{\mathbf{a}} \otimes \mathbf{h}$. Using item b) of Theorem 1.1.3 it is easy to check that $\pi_{*}$ is a map of complexes. Consider the filtration $F_{*}^{0 S} \subseteq F_{*}^{1 S} \subseteq F_{*}^{2 S} \subseteq \ldots$ of $\left(\bar{X}_{*}^{S}, \bar{d}_{*}^{S}\right)$, where $F_{n}^{i S}=\bigoplus_{0 \leq s \leq i}\left(M \otimes_{S} \widetilde{A}^{\left(\otimes_{S}\right)^{n-s}} \otimes_{S}\right) \otimes \bar{H}^{\otimes^{s}}$. From Theorem 1.2 of [G-S], it follows that $\pi_{*}$ is a morphism of filtered complexes inducing an quasiisomorphism between the graded complexes associated to the filtrations of $\left(\bar{X}_{*}, \bar{d}_{*}\right)$ and $\left(\bar{X}_{*}^{S}, \bar{d}_{*}^{S}\right)$. Consequently $\pi_{*}$ is a quasi-isomorphism. The proof can be finished by applying Theorem 3.1.1.

\subsection{Hochschild cohomology}

Let $\bar{X}^{r s}=\operatorname{Hom}_{k}\left(\bar{A}^{\otimes^{r}} \otimes \bar{H}^{\otimes^{s}}, M\right)$. The map $\theta^{r s}: \bar{X}^{r s} \rightarrow \widehat{X}^{r s}$, defined by $\theta^{r s}(\varphi)\left(\left(1 \# h_{1}\right) \otimes_{A} \cdots \otimes_{A}\left(1 \# h_{s}\right) \otimes \mathbf{a}\right)=\left(1 \# h_{1}^{(1)}\right) \cdots\left(1 \# h_{s}^{(1)}\right) \varphi\left(\mathbf{a} \otimes h_{1}^{(2)} \otimes \cdots \otimes h_{s}^{(2)}\right)$,

is an isomorphism. Let $\bar{d}_{l}^{r s}: \bar{X}^{r+l-1, s-l} \rightarrow \bar{X}^{r s}$ be the map defined by $\bar{d}_{l}^{r s}:=$ $\left(\theta^{r s}\right)^{-1} \widehat{d}_{l}^{r s} \theta^{r+l-1, s-l}$.

Theorem 3.2.1. The Hochschild cohomology of $E$ with coefficients in $M$ is the cohomology of $\left(\bar{X}^{*}, \bar{d}^{*}\right)$, where

$$
\bar{X}^{n}=\bigoplus_{r+s=n} \bar{X}^{r s} \quad \text { and } \quad \bar{d}^{n}:=\sum_{l=1}^{n} \bar{d}_{l}^{0 n}+\sum_{r=1}^{n} \sum_{l=0}^{n-r} \bar{d}_{l}^{r, n-r} .
$$

Proof. This is an immediate consequence of Theorem 2.4 and the fact that the map $\theta^{*}:\left(\bar{X}^{*}, \bar{d}^{*}\right) \rightarrow\left(\widehat{X}^{*}, \widehat{d}^{*}\right)$, given by $\theta^{n}=\sum_{r+s=n} \theta^{r s}$, is an isomorphism.

Note that when $f$ takes its values in $k$, then $\left(\bar{X}^{*}, \bar{d}^{*}\right)$ is the total complex of the double complex $\left(\operatorname{Hom}_{k}\left(\bar{A}^{*} \otimes \bar{H}^{*}, M\right), \bar{d}_{0}^{* *}, \bar{d}_{1}^{* *}\right)$. 
It is easy to see that

$$
\begin{aligned}
\bar{d}_{0}^{r s}(\varphi)(\mathbf{x}) & =a_{1} \varphi\left(\mathbf{a}_{2 r} \otimes \mathbf{h}\right)+(-1)^{r} \varphi\left(\mathbf{a}_{1, r-1} \otimes \mathbf{h}\right) a_{r} \\
& +\sum_{i=1}^{r-1}(-1)^{i} \varphi\left(\mathbf{a}_{1, i-1} \otimes a_{i} a_{i+1} \otimes \mathbf{a}_{i+2, r} \otimes \mathbf{h}\right), \\
\bar{d}_{1}^{r s}(\varphi)(\mathbf{x}) & =(-1)^{r} \epsilon\left(h_{1}\right) \varphi\left(\mathbf{a} \otimes \mathbf{h}_{2 s}\right)+(-1)^{r+s}\left(1 \# h_{s}^{(1)}\right)^{-1} \varphi\left(\mathbf{a}^{h_{s}^{(2)}} \otimes \mathbf{h}_{1, s-1}\right)\left(1 \# h_{s}^{(3)}\right) \\
& +\sum_{i=1}^{s-1}(-1)^{r+i} \varphi\left(\mathbf{a} \otimes \mathbf{h}_{1, i-1} \otimes h_{i} h_{i+1} \otimes \mathbf{h}_{i+2, s}\right),
\end{aligned}
$$

where $\mathbf{x}=\mathbf{a} \otimes \mathbf{h}$, with $\mathbf{a}=a_{1} \otimes \cdots \otimes a_{r}$ and $\mathbf{h}=h_{1} \otimes \cdots \otimes h_{s}$.

For each $h \in H$ we have the map $\vartheta_{h}^{*}:\left(\operatorname{Hom}_{k}\left(\bar{A}^{*}, M\right), b^{*}\right) \rightarrow\left(\operatorname{Hom}_{k}\left(\bar{A}^{*}, M\right), b^{*}\right)$, defined by $\vartheta_{h}^{r}(\varphi)(\mathbf{a})=\left(1 \# h^{(1)}\right)^{-1} \varphi\left(\mathbf{a}^{h^{(2)}}\right)\left(1 \# h^{(3)}\right)$.

Proposition 3.2.2. For each $h, l \in H$, the endomorphisms of $\mathrm{H}^{*}(A, M)$ induced by $\vartheta_{l}^{*} \vartheta_{h}^{*}$ and by $\vartheta_{h l}^{*}$ coincide. Consequently $\mathrm{H}^{*}(A, M)$ is a right $H$-module.

Proof. By a standard argument it is sufficient to prove it for $\mathrm{H}^{0}(A, M)$, and in this case the result is immediate.

Let $F_{0}\left(\bar{X}^{*}\right) \supseteq F_{1}\left(\bar{X}^{*}\right) \subseteq \ldots$ be the filtration of $\left(\bar{X}^{*}, \bar{d}^{*}\right)$ obtained transporting the one of $\left(\widehat{X}_{*}, \widehat{d}_{*}\right)$, given above Corollary 2.5 , through $\theta^{*}$. It is immediate that $F_{i}\left(\bar{X}_{n}\right)=\bigoplus_{s \geq i} \bar{X}^{n-s, s}$. Then, we have the following:

Corollary 3.2.3. if $H$ is flat over $k$, then the spectral sequence of Corollary 2.6 satisfies $E_{1}^{r s}=\operatorname{Hom}_{k}\left(\bar{H}^{s}, \mathrm{H}^{r}(A, M)\right)$ and $E_{2}^{r s}=\mathrm{H}^{s}\left(H, \mathrm{H}^{r}(A, M)\right)$.

Let $S$ be a separable subalgebra of $A$ and let $\widetilde{A}^{\left(\otimes_{S}\right)^{r}}(r \geq 0)$ be as in 3.1.4. Suppose $f(h, l) \in S$ for all $h, l \in H$. Using the fact that $f$ takes its values in $S$ it is easy to see that $H$ acts on $\left(\operatorname{Hom}_{A^{e}}\left(A \otimes_{S} \widetilde{A}^{\left(\otimes_{S}\right)^{r}} \otimes_{S} A, M\right), b^{*}\right)=\left(\operatorname{Hom}_{S^{e}}\left(\widetilde{A}^{\left(\otimes_{S}\right)^{r}}, M\right), b^{*}\right)$ $\operatorname{via}(\varphi \cdot h)(\widetilde{\mathbf{a}})=\left(1 \# h^{(1)}\right)^{-1} \varphi\left(\widetilde{\mathbf{a}}^{h^{(2)}}\right)\left(1 \# h^{(3)}\right)$.

Theorem 3.2.4. The Hochschild cohomology $\mathrm{H}^{*}(E, M)$, of $E$ with coefficients in $M$, is the cohomology of $H$ with coefficients in $\left(\operatorname{Hom}_{S^{e}}\left(\widetilde{A}^{\left(\otimes_{S}\right)^{r}}, M\right), b^{*}\right)$.

Proof. It is similar to the proof of Theorem 3.1.4.

\section{The Cartan-Leray and Grothendieck spectral sequences}

Assume that $E$ is a crossed product with invertible cocycle. In this case another two spectral sequences converging to $\mathrm{H}_{*}(E, M)$ and with $E^{2}$-term $\mathrm{H}_{*}\left(H, \mathrm{H}_{*}(A, M)\right)$ can be considered. They are the Cartan-Leray and the Grothendieck spectral sequences. The last one was introduced for the more general setting of Galois extension in $[\mathrm{S}]$. In this Section we recall these constructions and we prove that both coincide with the Hochschild-Serre spectral sequence. Similar results are valid in the cohomological setting. Recall that $\left(\mathrm{B}_{*}(E), b_{*}^{\prime}\right)$ is the normalized Hochschild resolution of $E$.

Let $\left(\bar{H}^{\otimes^{*}} \otimes H, d_{*}\right)$ be the canonical resolution of $k$ as a right $H$-module and $\left(Z_{*}, \partial_{*}\right)=\left(\mathrm{B}_{*}(E), b_{*}^{\prime}\right) \otimes\left(\bar{H}^{\otimes^{*}} \otimes H, d_{*}\right)$. Consider $E \otimes \bar{E}^{\otimes^{r}} \otimes E \otimes \bar{H}^{\otimes^{s}} \otimes H$ as an $E$-bimodule via

$$
(a \# l)(\mathbf{x} \otimes \mathbf{h})(b \# q)=\left((a \# l) x_{0} \otimes \mathbf{x}_{1 r} \otimes x_{r+1}\left(b \# q^{(1)}\right)\right) \otimes\left(\mathbf{h}_{1 s} \otimes h_{s+1} q^{(2)}\right),
$$


where $\mathbf{x}=x_{0} \otimes \cdots \otimes x_{r+1}$ and $\mathbf{h}=h_{1} \otimes \cdots \otimes h_{s+1}$. It is clear that

$$
E \stackrel{\mu}{\longleftarrow} Z_{0} \stackrel{\partial_{1}}{\longleftarrow} Z_{1} \stackrel{\partial_{2}}{\longleftarrow} Z_{2} \stackrel{\partial_{3}}{\longleftarrow} Z_{3} \stackrel{\partial_{4}}{\longleftarrow} Z_{4} \stackrel{\partial_{5}}{\longleftarrow} Z_{5} \stackrel{\partial_{6}}{\longleftarrow} \ldots
$$

where $\mu\left(\left(a_{0} \# h_{0} \otimes a_{1} \# h_{1}\right) \otimes l\right)=\epsilon(l) a_{0} a_{1} f\left(h_{0}^{(1)}, h_{1}^{(1)}\right) \# h_{0}^{(2)} h_{1}^{(2)}$, is a complex of $E$-bimodules. Moreover (3) is contractible as a complex of left $E$-modules, with contracting homotopy $\zeta_{n}(n \geq 0)$ given by $\zeta_{0}\left(1_{E}\right)=1_{E} \otimes 1_{E} \otimes 1_{H}$ and

$$
\zeta_{n+1}(\mathbf{y})=\left\{\begin{array}{ll}
-\mathbf{x} \otimes 1_{E} \otimes \mathbf{h}+(-1)^{n+1} x_{0} x_{1} \otimes 1_{E} \otimes \mathbf{h} \otimes 1_{H} & \text { if } r=0 \\
(-1)^{r+1} \mathbf{x} \otimes 1_{E} \otimes \mathbf{h} & \text { if } r>0
\end{array},\right.
$$

where $\mathbf{y}=\mathbf{x} \otimes \mathbf{h}$, with $\mathbf{x}=x_{0} \otimes \cdots \otimes x_{r+1}$ and $\mathbf{h}=h_{1} \otimes \cdots \otimes h_{n-r+1}$. Since the map

$$
\tau: E \otimes \bar{E}^{\otimes^{r}} \otimes \bar{H}^{\otimes^{s}} \otimes H \otimes E \rightarrow E \otimes \bar{E}^{\otimes^{r}} \otimes E \otimes \bar{H}^{\otimes^{s}} \otimes H,
$$

given by $\tau\left(\mathbf{x}_{0 r} \otimes \mathbf{h} \otimes x_{r+1}\right)=\left(\mathbf{x}_{0 r} \otimes 1_{E} \otimes \mathbf{h}\right) x_{r+1}$, is an isomorphism of $E$-bimodules (the inverse of $\tau$ is the map $\mathbf{x}_{0 r} \otimes a \# h \otimes \mathbf{h} \mapsto \mathbf{x}_{0 r} \otimes \mathbf{h}_{1 s} \otimes h_{s+1} S^{-1}\left(h^{(2)}\right) \otimes a \# h^{(1)}$ ), $\left(Z_{*}, \partial_{*}\right)$ is an $\Upsilon$-projective resolution of $E$.

We consider $\left(Z_{*}, \partial_{*}\right)$ filtered by $F^{0}\left(Z_{*}\right) \subseteq F^{1}\left(Z_{*}\right) \subseteq F^{2}\left(Z_{*}\right) \subseteq \ldots$, where $\left.F^{i}\left(Z_{n}\right)=\bigoplus_{j=0}^{i} E \otimes \bar{E}^{\otimes^{n-j}} \otimes E\right) \otimes\left(\bar{H}^{\otimes^{j}} \otimes H\right)$.

Let $M$ be an $E$-bimodule. The groups $M \otimes_{E \otimes A^{\text {op }}}\left(E \otimes \bar{E}^{\otimes^{r}} \otimes E\right)$ are left $H$ modules via

$$
h(m \otimes \mathbf{x})=\left(1 \# h^{(2)}\right) m \otimes \mathbf{x}_{0 r} \otimes x_{r+1}\left(1 \# h^{(1)}\right)^{-1},
$$

where $\mathbf{x}=x_{0} \otimes \cdots \otimes x_{r+1}$. There is an isomorphism

$$
M \otimes_{E^{e}}\left(Z_{*}, \partial_{*}\right) \simeq\left(\bar{H}^{\otimes^{*}} \otimes H, d_{*}\right) \otimes_{H}\left(M \otimes_{E \otimes A^{\text {op }}}\left(\mathrm{B}_{*}(E), b_{*}^{\prime}\right)\right) .
$$

Let $F_{*}^{0} \subseteq F_{*}^{1} \subseteq F_{*}^{2} \subseteq F_{*}^{3} \subseteq \ldots$ be the filtration of $M \otimes_{E^{e}}\left(Z_{*}, \partial_{*}\right)$ induced by the filtration of $\left(Z_{*}, \partial_{*}\right)$. It is easy to see that $F_{n}^{i} \simeq \bigoplus_{j=0}^{i}\left(\bar{H}^{\otimes^{j}} \otimes H\right) \otimes_{H}\left(M \otimes_{A \otimes E^{\text {op }}}\right.$ $\left.E \otimes \bar{E}^{\otimes^{n-j}} \otimes E\right)$. The spectral sequence associated to this filtration converges to $\mathrm{H}_{*}(E, M)$ and has $E^{2}$-term $\mathrm{H}_{*}\left(H, \mathrm{H}_{*}(A, M)\right)$. This spectral sequence is called the homological Cartan-Leray spectral sequence. Similarly the groups $\operatorname{Hom}_{E \otimes A^{\text {op }}}(E \otimes$ $\left.\bar{E}^{\otimes^{r}} \otimes E, M\right)$ are right $H$ modules via $f . h(\mathbf{x})=f\left(\mathbf{x}_{0 r} \otimes x_{r+1}\left(1 \# h^{(1)}\right)^{-1}\right)\left(1 \# h^{(2)}\right)$ and there is an isomorphism

$$
\operatorname{Hom}_{E^{e}}\left(\left(Z_{*}, \partial_{*}\right), M\right) \simeq \operatorname{Hom}_{H}\left(\left(\bar{H}^{\otimes^{*}} \otimes H, d_{*}\right), \operatorname{Hom}_{E \otimes A^{\text {op }}}\left(\left(\mathrm{B}_{*}(E), b_{*}^{\prime}\right), M\right)\right) .
$$

Let $F_{0}^{*} \supseteq F_{1}^{*} \supseteq F_{2}^{*} \supseteq F_{3}^{*} \supseteq F_{4}^{*} \supseteq \ldots$ be the filtration of $\operatorname{Hom}_{E^{e}}\left(\left(Z_{*}, \partial_{*}\right), M\right)$ induced by the filtration of $\left(Z_{*}, \partial_{*}\right)$. It is easy to see that $F_{i}^{n}=\bigoplus_{j \geq i} \operatorname{Hom}_{H}\left(\bar{H}^{\otimes^{j}} \otimes\right.$ $\left.H, \operatorname{Hom}_{E \otimes A^{\circ \mathrm{p}}}\left(E \otimes \bar{E}^{\otimes^{n-j}} \otimes E, M\right)\right)$. The spectral sequence associated to this filtration converges to $\mathrm{H}^{*}(E, M)$ and has $E^{2}$-term $\mathrm{H}^{*}\left(H, \mathrm{H}^{*}(A, M)\right)$. This spectral sequence is called the cohomological Cartan-Leray spectral sequence.

Let $\Phi_{*}:\left(\mathrm{B}_{*}(E), b_{*}^{\prime}\right) \rightarrow\left(Z_{*}, \partial_{*}\right)$ and $\Psi_{*}:\left(Z_{*}, \partial_{*}\right) \rightarrow\left(\mathrm{B}_{*}(E), b_{*}^{\prime}\right)$ be the morphisms of $E$-bimodule complexes, recursively defined by

$$
\begin{aligned}
& \Phi_{0}\left(x \otimes 1_{E}\right)=x \otimes 1_{E} \otimes 1_{H}, \quad \Psi_{0}\left(x \otimes 1_{E} \otimes h\right)=\epsilon(h) x \otimes 1_{E}, \\
& \Phi_{n+1}\left(\mathbf{x} \otimes 1_{E}\right)=\zeta_{n+1} \Phi_{n} b_{n+1}^{\prime}\left(\mathbf{x} \otimes 1_{E}\right), \\
& \Psi_{n+1}\left(\mathbf{x} \otimes 1_{E} \otimes \mathbf{h}\right)=\xi_{n+1} \psi_{n} \partial_{n+1}\left(\mathbf{x} \otimes 1_{E} \otimes \mathbf{h}\right) .
\end{aligned}
$$


Proposition 4.1. It is true that $\Psi_{*} \Phi_{*}=i d_{*}$ and that $\Phi_{*} \Psi_{*}$ is homotopically equivalent to the identity map. The homotopy $\Phi_{*} \Psi_{*} \stackrel{\Omega_{*+1}}{\longrightarrow} i d_{*}$ is recursively defined by $\Omega_{1}\left(x \otimes 1_{E} \otimes h\right)=x \otimes 1_{E} \otimes h \otimes 1_{H}$ and

$$
\Omega_{n+1}\left(\mathbf{x} \otimes 1_{E} \otimes \mathbf{h}\right)=\zeta_{n+1}\left(\Phi_{n} \Psi_{n}-i d-\Omega_{n} \partial_{n}\right)\left(\mathbf{x} \otimes 1_{E} \otimes \mathbf{h}\right),
$$

for $\mathbf{x}=x_{0} \otimes \cdots \otimes x_{r}$ and $\mathbf{h}=h_{1} \otimes \cdots \otimes h_{n+1-r}$.

Proof. It is easy to see that $\Phi_{*}$ and $\Psi_{*}$ are morphisms of complexes. Arguing as in Proposition 1.2.1 we get that $\Omega_{*+1}$ is a homotopy from $\Phi_{*} \Psi_{*}$ to the identity map. It remains to prove that $\Psi_{*} \Phi_{*}=i d_{*}$. It is clear that $\Psi_{0} \Phi_{0}=i d_{0}$. Assume that $\Psi_{n} \Phi_{n}=i d_{n}$. Since $\Phi_{n+1}\left(E \otimes \bar{E}^{\otimes^{n}} \otimes k\right) \subseteq \sum_{r=0}^{n+1} E \otimes \bar{E}^{\otimes^{r}} \otimes k \otimes \bar{H}^{\otimes^{n+1-r}} \otimes H$, we have that on $E \otimes \bar{E}^{\otimes^{n}} \otimes k$

$$
\begin{aligned}
\Psi_{n+1} \Phi_{n+1} & =\xi_{n+1} \Psi_{n} \partial_{n+1} \Phi_{n+1}=\xi_{n+1} \Psi_{n} \partial_{n+1} \zeta_{n+1} \Phi_{n} b_{n+1}^{\prime} \\
& =\xi_{n+1} \Psi_{n} \Phi_{n} b_{n+1}^{\prime}-\xi_{n+1} \Psi_{n} \zeta_{n} \partial_{n} \Phi_{n} b_{n+1}^{\prime}=\xi_{n+1} b_{n+1}^{\prime}=i d_{n+1}
\end{aligned}
$$

Next, we consider the normalized Hochschild resolution $\left(\mathrm{B}_{*}(E), b_{*}^{\prime}\right)$ filtered as in Proposition 1.2 .2 and the resolution $\left(Z_{*}, \partial_{*}\right)$ filtered by $F^{0}\left(Z_{*}\right) \subseteq F^{1}\left(Z_{*}\right) \subseteq \ldots$, where $F^{i}\left(Z_{n}\right)=\bigoplus_{j=0}^{i}\left(E \otimes \bar{E}^{\otimes^{n-j}} \otimes E\right) \otimes\left(\bar{H}^{\otimes^{j}} \otimes H\right)$.

Proposition 4.2. We have that

$$
\begin{gathered}
\Phi_{n}\left(a_{0} \# h_{0} \otimes \cdots \otimes a_{n+1} \# h_{n+1}\right)=\sum_{j=0}^{n}(-1)^{j(n+1)}\left(a_{0} \# h_{0}\right)\left(a_{1} \# h_{1}^{(1)}\right) \ldots\left(a_{j} \# h_{j}^{(1)}\right) \\
\otimes\left(a_{j+1} \# h_{j+1}^{(1)}\right) \otimes \cdots \otimes\left(a_{n+1} \# h_{n+1}^{(1)}\right) \otimes h_{1}^{(2)} \otimes \cdots \otimes h_{j}^{(2)} \otimes h_{j+1}^{(2)} \cdots h_{n+1}^{(2)} .
\end{gathered}
$$

Consequently the map $\Phi_{*}$ preserves filtrations.

Proof. It follows by induction on $n$, using the recursive definition of $\Phi_{*}$.

Proposition 4.3. The map $\Phi_{*}$ induces a homotopy equivalence of E-bimodule complexes between the graded complexes associated to the filtrations of $\left(\mathrm{B}_{*}(E), b_{*}^{\prime}\right)$ and $\left(Z_{*}, \partial_{*}\right)$.

Proof. Note that

$$
\begin{aligned}
\frac{F^{s}\left(X_{*}\right)}{F^{s-1}\left(X_{*}\right)} & =\left(X_{* s}, d_{* s}^{0}\right)=\left(E \otimes \bar{H}^{\otimes^{s}} \otimes \bar{A}^{\otimes^{*}} \otimes E, d_{* s}^{0}\right), \\
\frac{F^{s}\left(Z_{*}\right)}{F^{s-1}\left(Z_{*}\right)} & =\left(\mathrm{B}_{*}(E), b_{*}^{\prime}\right) \otimes \bar{H}^{\otimes^{s}} \otimes H,
\end{aligned}
$$

where $d_{*, s}^{0}$ is the boundary map introduced in Subsection 1.1. By Proposition 1.2.2 it suffices to check that $\Phi_{*}=\Phi_{*} \phi_{*}$ induces a homotopy equivalence $\widetilde{\Phi}_{*}^{s}$ of $E$ bimodules complexes, from $\left(E \otimes \bar{H}^{\otimes^{s}} \otimes \bar{A}^{\otimes^{*}} \otimes E, d_{* s}^{0}\right)$ to $\left(\mathrm{B}_{*}(E), b_{*}^{\prime}\right) \otimes \bar{H}^{\otimes^{s}} \otimes H$. Let $Y_{s}$ and $\mu_{s}$ be as in Subsection 1.1 and $\widetilde{Y}_{s}=E \otimes \bar{H}^{\otimes^{s}} \otimes H$ endowed with the structure 
of $E$-bimodule given by $x_{0}\left(x_{1} \otimes \mathbf{h}\right) x_{2}=x_{0} x_{1} x_{2} \otimes \mathbf{h}$, where $\mathbf{h}=h_{0} \otimes \cdots \otimes h_{s+1}$. Consider the diagram
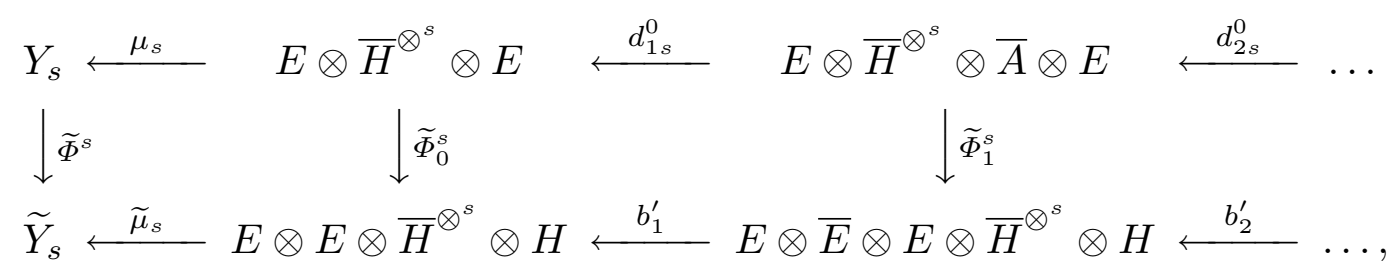

where

$$
\begin{aligned}
& \widetilde{\mu}_{s}\left(\left(x_{0} \otimes x_{1}\right) \otimes \mathbf{h}\right)=x_{0} x_{1} \otimes \mathbf{h} \quad \text { and } \\
& \widetilde{\Phi}^{s}(x \otimes \mathbf{h})=x\left(1 \# h_{1}^{(1)}\right) \cdots\left(1 \# h_{s+1}^{(1)}\right) \otimes h_{1}^{(2)} \otimes \cdots \otimes h_{s+1}^{(2)} .
\end{aligned}
$$

We assert that $\widetilde{\Phi}_{0}^{s}(\mathbf{x})=1_{E} \otimes\left(1 \# h_{1}^{(1)}\right) \cdots\left(1 \# h_{s}^{(1)}\right) \otimes h_{1}^{(2)} \otimes \cdots \otimes h_{s}^{(2)} \otimes 1_{H}$, where $\mathbf{x}=1_{E} \otimes \mathbf{h} \otimes 1_{E}$, with $\mathbf{h}=h_{1} \otimes \cdots \otimes h_{s}$. To prove this it suffices to check that

$$
\Phi_{s} \phi_{s}(\mathbf{x}) \in 1_{E} \otimes\left(1 \# h_{1}^{(1)}\right) \cdots\left(1 \# h_{s}^{(1)}\right) \otimes h_{1}^{(2)} \otimes \cdots \otimes h_{s}^{(2)} \otimes 1_{H}+F_{s-1}
$$

which follows by induction on $s$, using that $\Phi_{s} \phi_{s}(\mathbf{x})=\zeta_{s} \Phi_{s-1} \phi_{s-1} d_{s}(\mathbf{x})$. Now, it is immediate that $\widetilde{\mu}_{s} \widetilde{\Phi}_{0}^{s}=\widetilde{\Phi}^{s} \mu_{s}$. Since $\widetilde{\Phi}^{s}$ is an isomorphism and the rows of (4) are $\Upsilon$-projective resolutions of $Y_{s}$ and $\widetilde{Y}_{s}$ respectively, it follows that $\widetilde{\Phi}_{*}^{s}$ is a homotopy equivalence.

Corollary 4.4. The (co)homological Cartan-Leray spectral sequence is isomorphic to the (co)homological Hochschild-Serre spectral sequence.

Proof. We prove the assertion for the homology. The proof in the cohomological setting is similar. It is immediate that the filtration of $\left(M \otimes \bar{E}^{\otimes^{*}}, b_{*}\right)$ is the one induced by the filtration of $\left(\mathrm{B}_{*}(E), b_{*}^{\prime}\right)$, defined above Proposition 1.1.2. Hence, it is clear that the map $i d_{M} \otimes_{E^{e}} \Phi_{*}$ preserve filtrations. Let $\operatorname{gr}\left(i d_{M} \otimes_{E^{e}} \Phi_{*}\right)$ be the map induced by $i d_{M} \otimes_{E^{e}} \Phi_{*}$, between the graded complexes associated to the filtrations of $\left(M \otimes \bar{E}^{\otimes^{*}}, b_{*}\right)$ and $M \otimes_{E^{e}}\left(Z_{*}, \partial_{*}\right)$, respectively. Since, for all $i, n \geq 0$ the $E$-bimodules $F^{i}\left(\mathrm{~B}_{n}(E)\right)$ and $F^{i+1}\left(\mathrm{~B}_{n}(E)\right)$ are direct summands of $F^{i}\left(Z_{n}\right)$ and $F^{i+1}\left(Z_{n}\right)$ respectively, $\operatorname{gr}\left(i d_{M} \otimes_{E^{e}} \Phi_{*}\right)=i d_{M} \otimes_{E^{e}} \operatorname{gr}\left(\Phi_{*}\right)$, where $\operatorname{gr}\left(\Phi_{*}\right)$ is the map induced by $\Phi_{*}$, between the graded complexes associated to the filtrations of $\left(\mathrm{B}_{*}(E), b_{*}^{\prime}\right)$ and $\left(Z_{*}, \partial_{*}\right)$, respectively. By Proposition $4.3, \operatorname{gr}\left(i d_{M} \otimes_{E^{e}} \Phi_{*}\right)$ is a quasi-isomorphism. The assertion follows immediately from this fact.

4.5 The Grothendieck spectral sequence. If $M$ is an $E$-bimodule, then the group $\mathrm{H}_{0}(A, M)=M /[A, M]$ is a left $H$-module via $h \cdot \bar{m}=\overline{\left(1 \# h^{(2)}\right) m\left(1 \# h^{(1)}\right)^{-1}}$, where the $\bar{m}$ denotes the class of $m$ in $M /[A, M]$. Let us consider the functors $M \mapsto \mathrm{H}_{0}(E, M)$ from the category of $E$-bimodules to the category of $k$-modules, $M \mapsto \mathrm{H}_{0}(A, M)$ from the category of $E$-bimodules to the category of left $H$-modules and $M \mapsto \mathrm{H}_{0}(H, M)$ from the category of left $H$-modules to the category of $k$ modules. It is easy to see that $\mathrm{H}_{0}(E, M)=\mathrm{H}_{0}\left(H, \mathrm{H}_{0}(A, M)\right)$ and that if $M$ is a $\Upsilon$-projective module, then $\mathrm{H}_{0}(A, M)$ is a projective $H$-module, relative to the family of all epimorphisms of $H$-modules which split as $k$-linear maps. In fact, if $M=E \otimes N$, then the map $h \otimes n \mapsto \overline{1 \# h^{(2)} \otimes n\left(1 \# h^{(1)}\right)^{-1}}$ is an isomorphism of 
left $H$-modules from $H \otimes N$ to $\mathrm{H}_{0}(A, M)$. Thus we have a Grothendieck spectral sequence

$$
\left.E_{r s}^{2}=\mathrm{H}_{s}\left(H, \mathrm{H}_{r}(A, M)\right) \rightarrow \mathrm{H}_{r+s}(E, M)\right) .
$$

We assert that the Grothendieck spectral sequence and the Cartan-Leray spectral sequence coincide. To prove this we use a concrete construction of the Grothendieck spectral sequence. Let $\left(P_{*}, \partial_{*}\right)=\left(M \otimes \bar{E}^{\otimes^{*}} \otimes E, b_{*}^{\prime}\right)$ be the normalized canonical resolution of $M$ as a right $E$-module. Let us write $\left(\bar{P}_{*}, \bar{\partial}_{*}\right)=\left(P_{*}, \partial_{*}\right) \otimes_{A^{e}} A$. Consider the double complex

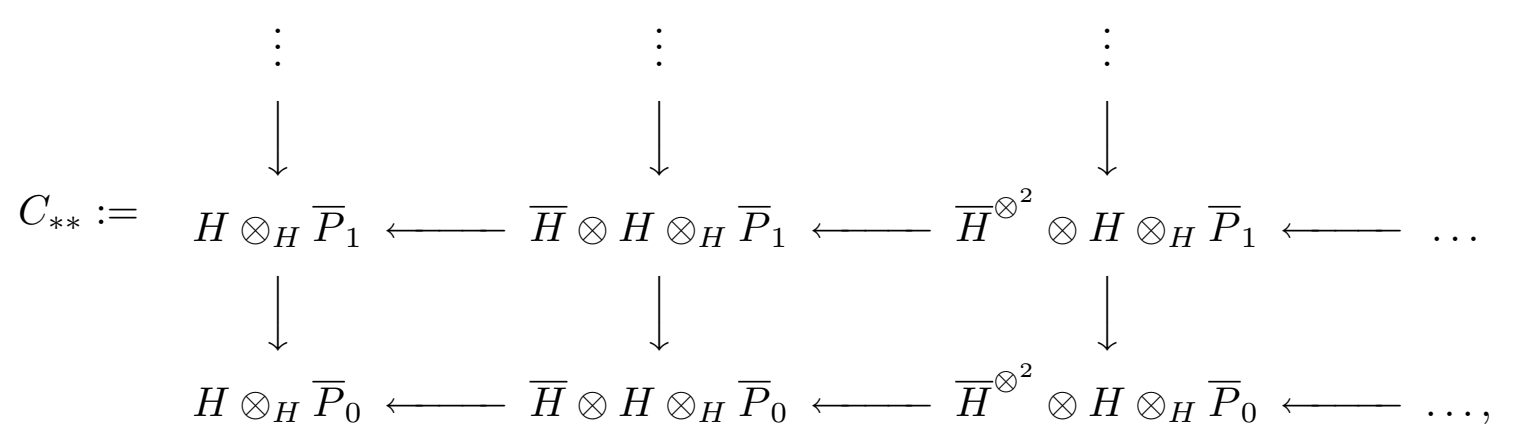

whose $r$-th column is $(-1)^{r}$ times $\bar{H}^{\otimes^{r}} \otimes H \otimes_{H}\left(\bar{P}_{*}, \bar{\partial}_{*}\right)$ and whose $s$-th row is the canonical complex $\left(\bar{H}^{\otimes^{*}} \otimes H \otimes_{H} \bar{P}_{s}, d_{*}\right)$ giving the homology $\mathrm{H}_{*}\left(H, \bar{P}_{s}\right)$ of $k$ as a trivial right $H$-module with coefficients in $\bar{P}_{s}$. By definition, the Grothendieck spectral sequence is the spectral sequence associated to the filtrations by columns of $C_{* *}$. Since $C_{* *} \simeq\left(\bar{H}^{\otimes^{*}} \otimes H, d_{*}\right) \otimes_{H}\left(M \otimes_{E \otimes A^{\text {op }}}\left(\mathrm{B}_{*}(E), b_{*}^{\prime}\right)\right)$ as filtered complexes, the homological Cartan-Leray and the Grothendieck spectral sequence coincide. The same is valid in the cohomological setting.

\section{Appendix A}

Let $R \rightarrow S$ be an unitary ring map and let $N$ be a left $S$-module. In this section, under suitable conditions, we construct a projective resolution of $N$, relative to the family of all epimorphisms of $S$-modules, which split as $R$-linear maps. We need this result (with $R=E, S=E^{e}$ and $N=E$ ) to complete the proof of Theorem 1.1.1. The general case considered here simplifies the notation and enables us to consider other cases, for instance algebras of groups having particular resolutions.

Let us consider a diagram of left $S$-modules and $S$-module maps

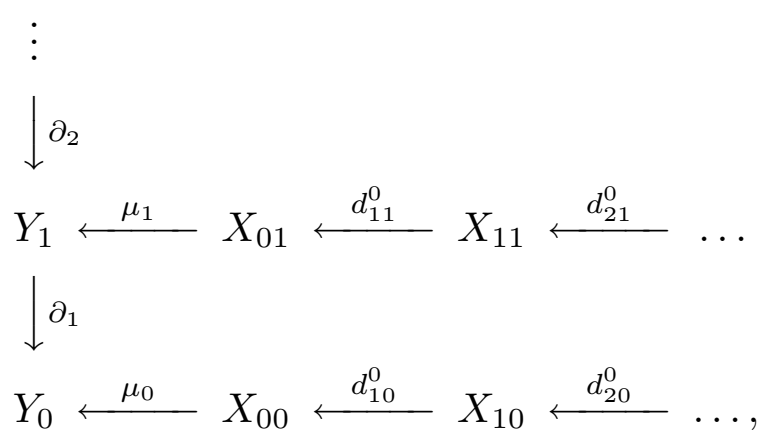

such that:

a) The column and the rows are chain complexes. 
b) For each $r, s \geq 0$ we have a left $R$-module $\bar{X}_{r s}$ and $S$-module maps

$$
s_{r s}: X_{r s} \rightarrow S \otimes \bar{X}_{r s} \quad \text { and } \quad \pi_{r s}: S \otimes \bar{X}_{r s} \rightarrow X_{r s}
$$

verifying $\pi_{r s} s_{r s}=i d$.

c) Each row is contractible as a complex of $R$-modules, with a chain contracting homotopy $\sigma_{0 s}^{0}: Y_{s} \rightarrow X_{0 s}$ and $\sigma_{r+1, s}^{0}: X_{r s} \rightarrow X_{r+1, s}(r \geq 0)$.

We are going to modify this diagram by adding $S$-module maps

$$
d_{r s}^{l}: X_{r s} \rightarrow X_{r+l-1, s-l} \quad(r, s \geq 0 \text { and } 1 \leq l \leq s) .
$$

For each $n \geq 0$, let

$$
X_{n}=\bigoplus_{r+s=n} X_{r s} \quad \text { and } \quad d_{n}=\sum_{l=1}^{n} d_{0 n}^{l}+\sum_{r=1}^{n} \sum_{l=0}^{n-r} d_{r, n-r}^{l} .
$$

Consider the maps $\mu_{n}^{\prime}: X_{n} \rightarrow Y_{n}(n \geq 0)$, given by:

$$
\mu_{n}^{\prime}(x)= \begin{cases}\mu_{n}(x) & \text { for } x \in X_{0 n} \\ 0 & \text { for } x \in X_{r, n-r}, \text { with } r>0 .\end{cases}
$$

We define the arrows $d_{r s}^{l}$ in such a way that $\left(X_{*}, d_{*}\right)$ becomes a chain complex of $S$-modules and $\mu_{*}^{\prime}:\left(X_{*}, d_{*}\right) \rightarrow\left(Y_{*},-\partial_{*}\right)$ becomes a chain homotopy equivalence of complexes of $R$-modules. In fact, we are going to build $R$-module morphisms

$$
\sigma_{l, s-l}^{l}: Y_{s} \rightarrow X_{l, s-l} \text { and } \sigma_{r+l+1, s-l}^{l}: X_{r s} \rightarrow X_{r+l+1, s-l} \quad(r, s \geq 0 \text { and } 1 \leq l \leq s) \text {, }
$$

satisfying the following:

Theorem A.1. Let $\mathrm{C}_{*}\left(\mu_{*}^{\prime}\right)$ be the mapping cone of $\mu_{*}^{\prime}$, that is, $\mathrm{C}_{*}\left(\mu_{*}^{\prime}\right)=\left(C_{*}, \delta_{*}\right)$, where $C_{n}=Y_{n} \oplus X_{n-1}$ and $\delta_{n}\left(y_{n}, x_{n-1}\right)=\left(-\partial\left(y_{n}\right)-\mu_{n-1}^{\prime}\left(x_{n-1}\right),-d_{n-1}\left(x_{n-1}\right)\right)$. The family of $R$-module maps $\sigma_{n+1}: \mathrm{C}_{n}\left(\mu_{*}^{\prime}\right) \rightarrow \mathrm{C}_{n+1}\left(\mu_{*}^{\prime}\right)(n \geq 0)$, defined by:

$$
\sigma_{n+1}=-\sum_{r=-1}^{n-1} \sum_{l=0}^{n-r-1} \sigma_{r+l+1, n-r-l-1}^{l}
$$

is a chain contracting homotopy of $\mathrm{C}_{*}\left(\mu_{*}^{\prime}\right)$.

Corollary A.2. Let $N$ be a left $S$-module. If there is an $S$-module map $\widetilde{\mu}: Y_{0} \rightarrow N$, such that

$$
N \stackrel{\widetilde{\mu}}{\longleftarrow} Y_{0} \stackrel{\partial_{1}}{\longleftarrow} Y_{1} \stackrel{\partial_{2}}{\longleftarrow} Y_{2} \stackrel{\partial_{3}}{\longleftarrow} Y_{3} \stackrel{\partial_{4}}{\longleftarrow} Y_{4} \stackrel{\partial_{5}}{\longleftarrow} Y_{6} \stackrel{\partial_{7}}{\longleftarrow} \ldots
$$

is contractible as a complex of left $R$-modules, then

$$
N \stackrel{\mu}{\longleftarrow} X_{0} \stackrel{d_{1}}{\longleftarrow} X_{1} \stackrel{d_{2}}{\longleftarrow} X_{2} \stackrel{d_{3}}{\longleftarrow} X_{3} \stackrel{d_{4}}{\longleftarrow} X_{4} \stackrel{d_{5}}{\longleftarrow} X_{5} \stackrel{d_{6}}{\longleftarrow} X_{6} \stackrel{d_{7}}{\longleftarrow} \ldots
$$

where $\mu=\widetilde{\mu} \mu_{0}$, is a relative projective resolution of $N$, relative to the family of all epimorphisms of $S$-modules, which split as $R$-linear maps. Moreover, if $\sigma_{0}^{-1}: N \rightarrow$ 
$Y_{0}, \sigma_{n+1}^{-1}: Y_{n} \rightarrow Y_{n+1}(n \geq 0)$ is a chain contracting homotopy of $\left(^{*}\right)$, then we obtain a chain contracting homotopy $\bar{\sigma}_{0}: N \rightarrow X_{0}, \bar{\sigma}_{n+1}: X_{n} \rightarrow X_{n+1}(n \geq 0)$ of $\left({ }^{* *}\right)$, defining $\bar{\sigma}_{0}=\sigma_{00}^{0} \sigma_{0}^{-1}$ and

$$
\bar{\sigma}_{n+1}=-\sum_{l=0}^{n+1} \sigma_{l, n-l+1}^{l} \sigma_{n+1}^{-1} \mu_{n}+\sum_{r=0}^{n} \sum_{l=0}^{n-r} \sigma_{r+l+1, n-r-l}^{l} .
$$

Proof. Write

$$
\widetilde{\sigma}_{n}=\sum_{r=0}^{n-1} \sum_{l=0}^{n-r-1} \sigma_{r+l+1, n-r-l-1}^{l} \quad(n \geq 1) \quad \text { and } \quad \widehat{\sigma}_{n}=\sum_{l=0}^{n} \sigma_{l, n-l}^{l} \quad(n \geq 0) .
$$

From Theorem A.1, we have

$$
\widehat{\sigma}_{n} \partial_{n+1}=\sum_{l=0}^{n} \sigma_{l, n-l}^{l} \partial_{n+1}=-\sum_{l=0}^{n} \sum_{i=0}^{l+1} d_{i, n+1-i}^{l+1-i} \sigma_{i, n+1-i}^{i}=-d_{n+1} \widehat{\sigma}_{n+1} .
$$

It is clear that $\mu \bar{\sigma}_{0}=i d$. Moreover

$$
\begin{aligned}
\bar{\sigma}_{0} \mu & =\sigma_{00}^{0} \sigma_{0}^{-1} \widetilde{\mu} \mu_{0}=\sigma_{00}^{0} \mu_{0}-\sigma_{00}^{0} \partial_{1} \sigma_{1}^{-1} \mu_{0} \\
& =i d-d_{10}^{0} \sigma_{10}^{0}+d_{01}^{1} \sigma_{01}^{0} \sigma_{1}^{-1} \mu_{0}+d_{10}^{0} \sigma_{10}^{1} \sigma_{1}^{-1} \mu_{0}
\end{aligned}
$$

where the last equality follows from $\left(*_{0}^{0}\right)$. Now, let $n \geq 1$. Take $x \in X_{r, n-r}$. If $r \geq 1$, then the equality $(0, x)=\delta_{n+2} \sigma_{n+2}(0, x)+\sigma_{n+1} \delta_{n+1}(0, x)$ implies that $x=d_{n+1} \widetilde{\sigma}_{n+1}(x)+\widetilde{\sigma}_{n} d_{n}(x)$. Hence, we can suppose $r=0$. Then, from $(0, x)=$ $\delta_{n+2} \sigma_{n+2}(0, x)+\sigma_{n+1} \delta_{n+1}(0, x)$, we get

$$
\begin{aligned}
x & =d_{n+1} \widetilde{\sigma}_{n+1}(x)+\widetilde{\sigma}_{n} d_{n}(x)+\widehat{\sigma}_{n} \mu_{n}(x) \\
& =d_{n+1} \widetilde{\sigma}_{n+1}(x)+\widetilde{\sigma}_{n} d_{n}(x)+\widehat{\sigma}_{n} \sigma_{n}^{-1} \partial_{n} \mu_{n}(x)+\widehat{\sigma}_{n} \partial_{n+1} \sigma_{n+1}^{-1} \mu_{n}(x) \\
& =d_{n+1} \widetilde{\sigma}_{n+1}(x)+\widetilde{\sigma}_{n} d_{n}(x)-\widehat{\sigma}_{n} \sigma_{n}^{-1} \mu_{n-1} d_{n}(x)+\widehat{\sigma}_{n} \partial_{n+1} \sigma_{n+1}^{-1} \mu_{n}(x) \\
& =d_{n+1} \widetilde{\sigma}_{n+1}(x)+\widetilde{\sigma}_{n} d_{n}(x)-\widehat{\sigma}_{n} \sigma_{n}^{-1} \mu_{n-1} d_{n}(x)-d_{n+1} \widehat{\sigma}_{n+1} \sigma_{n+1}^{-1} \mu_{n}(x),
\end{aligned}
$$

where the last equality follows from $\left({ }^{*} n\right)$.

Next we define the morphisms $d_{r s}^{l}$ and we prove that $\left(X_{*}, d_{*}\right)$ is a chain complex.

Definition A.3. We define the $S$-module maps $d_{r s}^{l}: X_{r s} \rightarrow X_{r+l-1, s-l}(r \geq 0$ and $1 \leq l \leq s)$, recursively by $d_{r s}^{l}=\bar{d}_{r s}^{l} s_{r s}$, where $\bar{d}_{r s}^{l}: S \otimes \bar{X}_{r s} \rightarrow X_{r+l-1, s-l}(r \geq 0$ and $1 \leq l \leq s$ ) is the $S$-module map defined by

$$
\bar{d}_{r s}^{l}(\mathbf{x})= \begin{cases}-\sigma_{0, s-1}^{0} \partial_{s} \mu_{s} \pi_{0 s}(\mathbf{x}) & \text { if } r=0 \text { and } l=1, \\ -\sum_{j=1}^{l-1} \sigma_{l-1, s-l}^{0} d_{j-1, s-j}^{l-j} d_{0 s}^{j} \pi_{0 s}(\mathbf{x}) & \text { if } r=0 \text { and } 1<l \leq s, \\ -\sum_{j=0}^{l-1} \sigma_{r+l-1, s-l}^{0} d_{r+j-1, s-j}^{l-j} d_{r s}^{j} \pi_{r s}(\mathbf{x}) & \text { if } r>0,\end{cases}
$$

for each $\mathbf{x}=1 \otimes \overline{\mathbf{x}} \in S \otimes \bar{X}_{r s}$. 
Proposition A.4. We have $\mu_{s-1} d_{0 s}^{1}=-\partial_{s} \mu_{s}$ and

$$
d_{r+l-1, s-l}^{0} d_{r s}^{l}= \begin{cases}-\sum_{j=1}^{l-1} d_{j-1, s-j}^{l-j} d_{0 s}^{j} & \text { if } r=0 \text { and } 1<l \leq s \\ -\sum_{j=0}^{l-1} d_{r+j-1, s-j}^{l-j} d_{r s}^{j} & \text { if } r>0 \text { and } 1 \leq l \leq s .\end{cases}
$$

Consequently $\left(X_{*}, d_{*}\right)$ is a chain complex.

Proof. We prove the proposition by induction on $l$ and $r$. To simplify the expressions we put $d_{0 s}^{0}:=\mu_{s}, d_{-1, s}^{1}:=\partial_{s}$ and $d_{-1, s}^{l}:=0$ for all $l>1$. Moreover to abbreviate we do not write the subindices. Let $\mathbf{x}=1 \otimes \overline{\mathbf{x}}$ with $\overline{\mathbf{x}} \in \bar{X}_{0 s}$. Since $\bar{d}_{0}^{1}(\mathbf{x})=-\sigma^{0} d^{1} d^{0} \pi(\mathbf{x})$, we have $d^{0} \bar{d}^{1}(\mathbf{x})=-d^{0} \sigma^{0} d^{1} d^{0} \pi(\mathbf{x})=-d^{1} d^{0} \pi(\mathbf{x})$, which implies $d^{0} d^{1}=-d^{1} d^{0}$. Let $l+r>1$ and suppose the result is valid for $d_{p *}^{j}$ with $j<l$ or $j=l$ and $p<r$. Let $\mathbf{x}=1 \otimes \overline{\mathbf{x}}$ with $\overline{\mathbf{x}} \in \bar{X}_{r s}$. Since $\bar{d}^{l}(\mathbf{x})=-\sum_{j=0}^{l-1} \sigma^{0} d^{l-j} d^{j} \pi(\mathbf{x})$, then

$$
d^{0} \bar{d}^{l}(\mathbf{x})=-\sum_{j=0}^{l-1} d^{0} \sigma^{0} d^{l-j} d^{j} \pi(\mathbf{x})=-\sum_{j=0}^{l-1} d^{l-j} d^{j} \pi(\mathbf{x})+\sum_{j=0}^{l-1} \sigma^{0} d^{0} d^{l-j} d^{j} \pi(\mathbf{x}) .
$$

Applying first the inductive hypothesis to $d^{0} d^{l-j}$ with $(0 \leq j<l)$ and then to $d^{0} d^{j}$ with $(0<j<l)$, we obtain:

$$
\begin{aligned}
d^{0} \bar{d}^{l}(\mathbf{x}) & =-\sum_{j=0}^{l-1} d^{l-j} d^{j} \pi(\mathbf{x})-\sum_{j=0}^{l-1} \sum_{i=0}^{l-j-1} \sigma^{0} d^{l-j-i} d^{i} d^{j} \pi(\mathbf{x}) \\
& =-\sum_{j=0}^{l-1} d^{l-j} d^{j} \pi(\mathbf{x})-\sum_{j=0}^{l-2} \sum_{i=1}^{l-j-1} \sigma^{0} d^{l-j-i} d^{i} d^{j} \pi(\mathbf{x}) \\
& +\sum_{j=1}^{l-1} \sum_{h=0}^{j-1} \sigma^{0} d^{l-j} d^{j-h} d^{h} \pi(\mathbf{x})=-\sum_{j=0}^{l-1} d^{l-j} d^{j} \pi(\mathbf{x}) .
\end{aligned}
$$

The desired equality follows immediately from this fact.

It is immediate that $\mu_{*}^{\prime}:\left(X_{*}, d_{*}\right) \rightarrow\left(Y_{*},-\partial_{*}\right)$ is a morphism of $S$-module chain complexes. Next, we construct the chain contracting homotopy of $\mathrm{C}_{*}\left(\mu_{*}^{\prime}\right)$.

Definition A.5. We define $\sigma_{l, s-l}^{l}: Y_{s} \rightarrow X_{l, s-l}$ and $\sigma_{r+l+1, s-l}^{l}: X_{r s} \rightarrow X_{r+l+1, s-l}$ $(0<l \leq s, r \geq 0)$, recursively by:

$$
\sigma_{r+l+1, s-l}^{l}=-\sum_{i=0}^{l-1} \sigma_{r+l+1, s-l}^{0} d_{r+i+1, s-i}^{l-i} \sigma_{r+i+1, s-i}^{i} \quad(0<l \leq s \text { and } r \geq-1) .
$$

Proof of Theorem A.1. To simplify the expressions we put $d_{-1, s}^{0}:=0, d_{0 s}^{0}:=\mu_{s}$, $d_{-1, s}^{1}:=\partial_{s}$ and $d_{-1, s}^{l}:=0$ for all $l>1$. Because of the definitions of $d_{*}$ and $\sigma_{*}$, it suffices to check that $\sigma_{r s}^{0} d_{r s}^{0}+d_{r+1, s}^{0} \sigma_{r+1, s}^{0}=i d$ and

$$
\sum_{i=0}^{l} \sigma_{r+l, s-l}^{l-i} d_{r s}^{i}+\sum_{i=0}^{l} d_{r+i+1, s-i}^{l-i} \sigma_{r+i+1, s-i}^{i}=0 \quad \text { for } l>0
$$


where we put $d_{-1, s}^{0}=0$. The first formula simply says that $\sigma_{*}^{0}$ is a chain contracting homotopy of $d_{*}^{0}$. Let us see the second one. To abbreviate we do not write the subindices. From the definition of $\sigma^{l}$ we have:

$$
d^{0} \sigma^{l}=-\sum_{i=0}^{l-1} d^{0} \sigma^{0} d^{l-i} \sigma^{i}=\sum_{i=0}^{l-1} \sigma^{0} d^{0} d^{l-i} \sigma^{i}-\sum_{i=0}^{l-1} d^{l-i} \sigma^{i} .
$$

Consequently

$$
\sum_{i=0}^{l} \sigma^{l-i} d^{i}+\sum_{i=0}^{l} d^{l-i} \sigma^{i}=\sum_{i=0}^{l} \sigma^{l-i} d^{i}+\sum_{i=0}^{l-1} \sigma^{0} d^{0} d^{l-i} \sigma^{i} .
$$

Then, it suffices to prove that the term appearing on the right side of the equality is zero. We prove this by induction on $l$. For $l=1$ we have:

$$
\sigma^{0} d^{0} d^{1} \sigma^{0}=-\sigma^{0} d^{1} d^{0} \sigma^{0}=\sigma^{0} d^{1} \sigma^{0} d^{0}-\sigma^{0} d^{1}=-\sigma^{1} d^{0}-\sigma^{0} d^{1} .
$$

Suppose $l>1$. From Proposition A.5,

$$
\sum_{i=0}^{l-1} \sigma^{0} d^{0} d^{l-i} \sigma^{i}=-\sum_{i=0}^{l-1} \sum_{j=0}^{l-i-1} \sigma^{0} d^{l-i-j} d^{j} \sigma^{i}=-\sum_{h=0}^{l-1} \sum_{i=0}^{h} \sigma^{0} d^{l-h} d^{h-i} \sigma^{i} .
$$

So, applying the inductive hypothesis to $\sum_{i=0}^{h} d^{h-i} \sigma^{i}(h \geq 0)$, we obtain

$$
\begin{aligned}
\sum_{i=0}^{l-1} \sigma^{0} d^{0} d^{l-i} \sigma^{i} & =\sum_{h=0}^{l-1} \sum_{i=0}^{h} \sigma^{0} d^{l-h} \sigma^{h-i} d^{i}-\sigma^{0} d^{l} \\
& =\sum_{i=0}^{l-1} \sum_{j=0}^{l-i-1} \sigma^{0} d^{l-i-j} \sigma^{j} d^{i}-\sigma^{0} d^{l} \\
& =-\sum_{i=0}^{l} \sigma^{l-i} d^{i} .
\end{aligned}
$$

\section{Appendix B}

In this appendix we compute explicitly the maps $d_{r s}^{l}$ introduced in Section 1, completing the results of Theorem 1.1.3. We use the following notations:

1) Given $h_{0} \otimes \cdots \otimes h_{s} \in H^{\otimes^{s+1}}$ and $0 \leq i<j \leq s$, we write $\mathfrak{h}_{i j}=h_{i} \cdots h_{j} \in H$.

2) Given $\mathbf{h}=h_{0} \otimes \cdots \otimes h_{s} \in H^{\otimes^{s+1}}$, we let $\mathbf{h}^{(1)} \otimes \mathbf{h}^{(2)}$ denote the comultiplication of $\mathbf{h}$ in $H^{\otimes^{s+1}}$. So, $\mathbf{h}^{(1)} \otimes \mathbf{h}^{(2)}=\left(h_{0}^{(1)} \otimes \cdots \otimes h_{s}^{(1)}\right) \otimes\left(h_{0}^{(2)} \otimes \cdots \otimes h_{s}^{(2)}\right)$.

3) Given $a \in A, \mathbf{a}=a_{1} \otimes \cdots \otimes a_{r} \in A^{\otimes^{r}}$ and $\mathbf{h}=h_{0} \otimes \cdots \otimes h_{s} \in H^{\otimes^{s+1}}$, we write $a^{\mathbf{h}}=\left(\ldots\left(\left(\left(a^{h_{s}}\right)^{h_{s-1}}\right)^{h_{s-2}}\right)^{h_{s-3}} \ldots\right)^{h_{0}}$ and $\mathbf{a}^{\mathbf{h}}=a_{1}^{\mathbf{h}^{(1)}} \otimes \cdots \otimes a_{r}^{\mathbf{h}^{(r)}}$.

Definition B.1. Given $\mathbf{h}=h_{1} \otimes \cdots \otimes h_{l} \in \bar{H}^{\otimes^{l}}$, we define $F_{0}^{(l)}(\mathbf{h}) \in \bar{A}^{\otimes^{l-1}}$, recursively by:

$$
\begin{aligned}
& F_{0}^{(2)}(\mathbf{h})=-f\left(h_{1}, h_{2}\right), \\
& F_{0}^{(l+1)}(\mathbf{h})=\sum_{j=1}^{l}(-1)^{j} f\left(h_{j}^{(1)}, h_{j+1}^{(1)}\right)^{\mathbf{h}_{1, j-1}^{(1)}} \otimes F^{(l)}\left(\mathbf{h}^{j(2)}\right),
\end{aligned}
$$


where $\mathbf{h}^{j(2)}=\mathbf{h}_{1, j-1}^{(2)} \otimes \mathrm{h}_{j}^{(2)} h_{j+1}^{(2)} \otimes \mathbf{h}_{j+2, l+1}$. For instance, we have

$$
F_{0}^{(3)}(\mathbf{h})=f\left(h_{1}^{(1)}, h_{2}^{(1)}\right) \otimes f\left(\mathfrak{h}_{12}^{(2)}, h_{3}\right)-f\left(h_{2}^{(1)}, h_{3}^{(1)}\right)^{h_{1}^{(1)}} \otimes f\left(h_{1}^{(2)}, \mathfrak{h}_{23}^{(2)}\right)
$$

and

$$
\begin{aligned}
F_{0}^{(4)}(\mathbf{h})= & -f\left(h_{1}^{(1)}, h_{2}^{(1)}\right) \otimes f\left(\mathfrak{h}_{12}^{(2)}, h_{3}^{(1)}\right) \otimes f\left(\mathfrak{h}_{12}^{(3)} h_{3}^{(2)}, h_{4}\right) \\
& +f\left(h_{1}^{(1)}, h_{2}^{(1)}\right) \otimes f\left(h_{3}^{(1)}, h_{4}^{(1)}\right)^{\mathfrak{h}_{12}^{(2)}} \otimes f\left(\mathfrak{h}_{12}^{(2)}, \mathfrak{h}_{34}^{(2)}\right) \\
& +f\left(h_{2}^{(1)}, h_{3}^{(1)}\right)^{h_{1}^{(1)}} \otimes f\left(h_{1}^{(2)}, \mathfrak{h}_{23}^{(2)}\right) \otimes f\left(\mathfrak{h}_{13}^{(3)}, h_{4}\right) \\
& -f\left(h_{2}^{(1)}, h_{3}^{(1)}\right)^{h_{1}^{(1)}} \otimes f\left(\mathfrak{h}_{23}^{(2)}, h_{4}^{(1)}\right)^{h_{1}^{(2)}} \otimes f\left(h_{1}^{(3)}, \mathfrak{h}_{23}^{(3)} h_{4}^{(2)}\right) \\
& -f\left(h_{3}^{(1)}, h_{4}^{(1)}\right)^{\mathbf{h}_{12}^{(1)}} \otimes f\left(h_{1}^{(2)}, h_{2}^{(2)}\right) \otimes f\left(\mathfrak{h}_{12}^{(3)}, \mathfrak{h}_{34}^{(2)}\right) \\
& +f\left(h_{3}^{(1)}, h_{4}^{(1)}\right)^{\mathbf{h}_{12}^{(1)}} \otimes f\left(h_{2}^{(2)}, \mathfrak{h}_{34}^{(2)}\right)^{h_{1}^{(2)}} \otimes f\left(h_{1}^{(3)}, \mathfrak{h}_{24}^{(3)}\right) .
\end{aligned}
$$

For the following definition we adopt the convention that $\mathbf{a}_{10}=\mathbf{a}_{r+1, r}=1_{k} \in k$.

Definition B.2. Given $\mathbf{h}=h_{1} \otimes \cdots \otimes h_{l} \in \bar{H}^{\otimes^{l}}$ and $\mathbf{a}=a_{1} \otimes \cdots \otimes a_{r} \in \bar{A}^{\otimes^{r}}$, we define $F_{r}^{(l)}(\mathbf{h} \otimes \mathbf{a})$, recursively by:

$$
\begin{aligned}
& F_{r}^{(2)}(\mathbf{h} \otimes \mathbf{a})=\sum_{i=0}^{r}(-1)^{i+1} \mathbf{a}_{1 i}^{\mathbf{h}_{12}^{(1)}} \otimes f\left(h_{1}^{(2)}, h_{2}^{(2)}\right) \otimes \mathbf{a}_{i+1, r}^{\mathfrak{h}_{12}^{(3)}}, \\
& F_{r}^{(l+1)}(\mathbf{h} \otimes \mathbf{a})=\sum_{j=1}^{l} \sum_{i=0}^{r}(-1)^{i l+j} \mathbf{a}_{1 i}^{(1)}{ }^{(1)}{ }^{r}\left(h_{j}^{(2)}, h_{j+1}^{(2)}\right)^{\mathbf{h}_{1, j-1}^{(2)}} \otimes F_{r-i}^{(l)}\left(\mathbf{h}^{j(3)} \otimes \mathbf{a}_{i+1, r}\right),
\end{aligned}
$$

where $\mathbf{h}^{j(3)}=\mathbf{h}_{1, j-1}^{(3)} \otimes \mathbf{h}_{j}^{(3)} h_{j+1}^{(3)} \otimes \mathbf{h}_{j+2, l+1}^{(2)}$ and $F_{0}^{(l)}\left(\mathbf{h}^{j(3)} \otimes \mathbf{a}_{r+1, r}\right)=F_{0}^{(l)}\left(\mathbf{h}^{j(3)}\right)$. For instance, we have

$$
\begin{aligned}
F_{r}^{(3)}(\mathbf{h} \otimes \mathbf{a})=\sum_{0 \leq i \leq j \leq r}(-1)^{i+j} \mathbf{a}_{1 i}^{\mathbf{h}_{13}^{(1)}} \otimes f\left(h_{1}^{(2)}, h_{2}^{(2)}\right) \otimes \mathbf{a}_{i+1, j}^{\mathbf{h}_{13}^{1(3)}} \otimes f\left(\mathfrak{h}_{12}^{(4)}, h_{3}^{(3)}\right) \otimes \mathbf{a}_{j+1, r}^{\mathfrak{h}_{12}^{(5)} h_{3}^{(4)}} \\
+\sum_{0 \leq i \leq j \leq r}(-1)^{i+j+1} \mathbf{a}_{1 i}^{\mathbf{h}_{13}^{(1)}} \otimes f\left(h_{2}^{(2)}, h_{3}^{(2)}\right)^{h_{1}^{(2)}} \otimes \mathbf{a}_{i+1, j}^{\mathbf{h}_{13}^{2(3)}} \otimes f\left(h_{1}^{(4)}, \mathfrak{h}_{23}^{(4)}\right) \otimes \mathbf{a}_{j+1, r}^{\mathfrak{h}_{13}^{(5)}} .
\end{aligned}
$$

We set $F_{0}^{(1)}\left(h_{s}\right)=1_{k} \in k, F_{r}^{(1)}\left(h_{s} \otimes \mathbf{a}\right)=\mathbf{a}^{h_{s}}$ and $F_{0}^{(l)}\left(\mathbf{h}_{s-l-1, s} \otimes 1_{k}\right)=$ $F_{0}^{(l)}\left(\mathbf{h}_{s-l-1, s}\right)$. Moreover, to abbreviate we write $F^{(l)}(\mathbf{h})=F_{0}^{(l)}(\mathbf{h})$ and $F^{(l)}\left(\begin{array}{c}\mathbf{a} \\ \mathbf{h}\end{array}\right)=$ $F_{r}^{(l)}(\mathbf{h} \otimes \mathbf{a})$.

Lemma B.3. Let $\mathbf{a}=a_{1} \otimes \cdots \otimes a_{r}$ and $\mathbf{h}_{s-l, s}=h_{s-l} \otimes \cdots \otimes h_{l}$. We have:

$$
F^{(l+1)}\left(\mathbf{h}_{s-l, s}\right)=\sum_{i=1}^{l}(-1)^{i} F^{(l-i+1)}\left(\begin{array}{c}
F^{(i)}\left(\mathbf{h}_{s-i+1, s}^{(1)}\right) \\
\mathbf{h}_{s-l, s-i}^{(1)}
\end{array}\right) \otimes f\left(\mathfrak{h}_{s-l, s-i}^{(2)}, \mathfrak{h}_{s-i+1, s}^{(2)}\right)
$$


and

$$
\begin{aligned}
F^{(l+1)}\left(\begin{array}{c}
\mathbf{a} \\
\mathbf{h}_{s-l, s}
\end{array}\right) & =F^{(l+1)}\left(\begin{array}{c}
\mathbf{a}_{1, r-1} \\
\mathbf{h}_{s-l, s}^{(1)}
\end{array}\right) \otimes a_{r}^{\mathfrak{h}_{s-l, s}^{(2)}} \\
& +\sum_{i=1}^{l}(-1)^{r+i} F^{(l-i+1)}\left(\begin{array}{c}
F^{(i)} \\
\mathbf{h}_{s-i+1, s}^{(1)} \\
\mathbf{h}_{s-l, s-i}^{(1)}
\end{array}\right) \otimes f\left(\mathfrak{h}_{s-l, s-i}^{(2)}, \mathfrak{h}_{s-i+1, s}^{(2)}\right)
\end{aligned}
$$

where $F^{(l+1)}\left(\begin{array}{l}\mathbf{a}_{1, r-1} \\ \mathbf{h}_{s-l, s}\end{array}\right)=F^{(l+1)}\left(\mathbf{h}_{s-l, s}\right)$ if $r=1$.

Proof. We prove the second formula. The proof of the first one is similar. It is clear that the lemma is valid for $l=1$. Let $l>1$ and suppose the result is valid for $l-1$. To abbreviate we put

$$
\begin{aligned}
& \xi=u(l-1)+j+s \\
& \mathfrak{h}_{s-l, s}^{j(4)}=\mathfrak{h}_{s-l, j+1}^{(4)} \mathfrak{h}_{j+2, s}^{(3)}, \\
& \mathbf{h}_{s-l, s}^{j(3)}=\mathbf{h}_{s-l, j-1}^{(3)} \otimes h_{j}^{(3)} h_{j+1}^{(3)} \otimes \mathbf{h}_{j+2, s}^{(2)}, \\
& \mathbf{f}_{j}^{(2)}=f\left(h_{j}^{(2)}, h_{j+1}^{(2)}\right)^{(2)} \mathbf{h}_{s-l, j-1}^{(2)}, \\
& \mathbf{f}_{s-l, s-i, s}^{j(4)}=f\left(\mathfrak{h}_{s-l, j+1}^{(4)} \mathfrak{h}_{j+2, s-i}^{(3)}, \mathfrak{h}_{s-i+1, s}^{(3)}\right), \\
& \mathbf{f}_{s-l, s-i, s}^{(4) j}=f\left(\mathfrak{h}_{s-l, s-i}^{(4)}, \mathfrak{h}_{s-i+1, j+1}^{(4)} \mathfrak{h}_{j+2, s}^{(3)}\right) \\
& \mathbf{f}_{s-l, s-i, s}^{(2)}=f\left(\mathfrak{h}_{s-l, s-i}^{(2)}, \mathfrak{h}_{s-i+1, s}^{(2)}\right) .
\end{aligned}
$$

We have:

$$
\begin{aligned}
& F^{(l+1)}\left(\begin{array}{c}
\mathbf{a} \\
\mathbf{h}_{s-l, s}
\end{array}\right)=\sum_{j=s-l}^{s-1} \sum_{u=0}^{r}(-1)^{\xi-1} \mathbf{a}_{1 u}^{\mathbf{h}_{s-l, s}^{(1)}} \otimes \mathbf{f}_{j}^{(2)} \otimes F^{(l)}\left(\begin{array}{c}
\mathbf{a}_{u+1, r} \\
\mathbf{h}_{s-l, s}^{j(3)}
\end{array}\right) \\
& =\sum_{j=s-l}^{s-1} \sum_{u=0}^{r-1}(-1)^{\xi-1} \mathbf{a}_{1 u}^{\mathbf{h}_{s-l, s}^{(1)}} \otimes \mathbf{f}_{j}^{(2)} \otimes F^{(l)}\left(\begin{array}{c}
\mathbf{a}_{u+1, r-1} \\
\mathbf{h}_{s-l, s}^{j(3)}
\end{array}\right) \otimes a_{r}^{\mathfrak{h}_{s-l, s}^{j(4)}}
\end{aligned}
$$

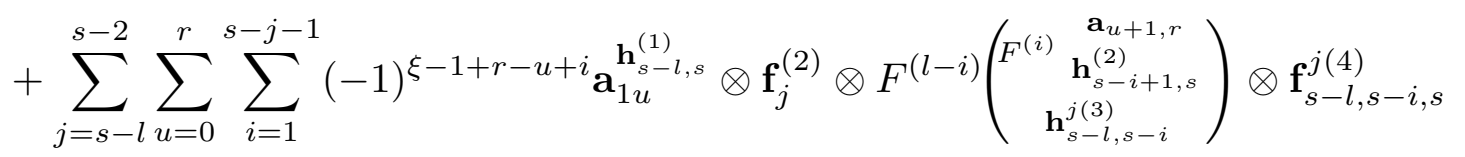

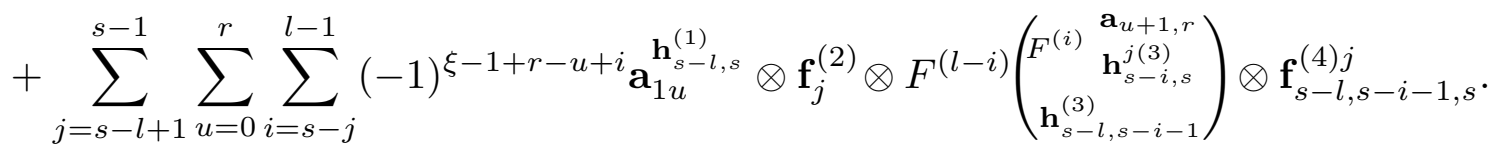

Permuting the order of the summands, we obtain

$$
\begin{aligned}
& F^{(l+1)}\left(\begin{array}{c}
\mathbf{a} \\
\mathbf{h}_{s-l, s}
\end{array}\right)=\sum_{j=s-l}^{s-1} \sum_{u=0}^{r-1}(-1)^{\xi-1} \mathbf{a}_{1 u}^{\mathbf{h}_{s-l, s}^{(1)}} \otimes \mathbf{f}_{j}^{(2)} \otimes F^{(l)}\left(\begin{array}{c}
\mathbf{a}_{u+1, r-1} \\
\mathbf{h}_{s-l, s}^{j(3)}
\end{array}\right) \otimes a_{r}^{\mathfrak{h}_{s-l, s}^{j(4)}}
\end{aligned}
$$

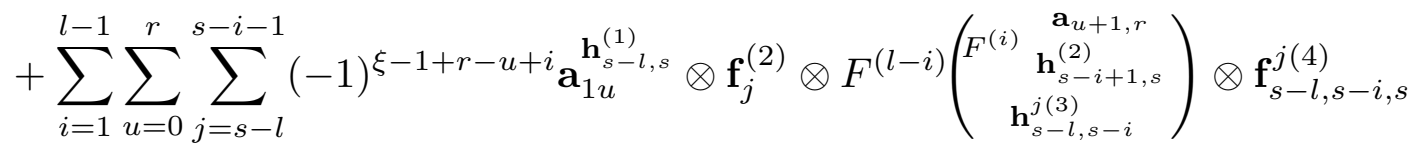

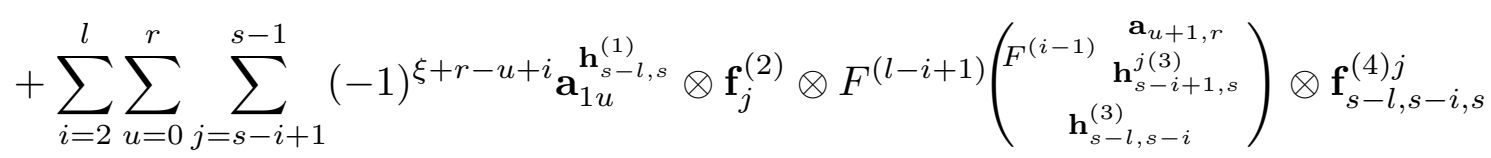




$$
=F^{(l+1)}\left(\begin{array}{l}
\mathbf{a}_{1, r-1} \\
\mathbf{h}_{s-l, s}^{(1)}
\end{array}\right) \otimes a_{r}^{\mathfrak{h}_{s-l, s}^{(2)}}+\sum_{i=1}^{l}(-1)^{r+i} F^{(l-i+1)}\left(\begin{array}{c}
F^{(i)} \\
\mathbf{h}_{s-i+1, s}^{(1)} \\
\mathbf{h}_{1 r}^{(1)} \\
\mathbf{h}_{s-l, s-i}
\end{array}\right) \otimes \mathbf{f}_{s-l, s-i, s}^{(2)},
$$

which ends the proof.

In the following theorem we identify $X_{r s}$ with $E \otimes \bar{H}^{\otimes^{s}} \otimes \bar{A}^{\otimes^{r}} \otimes E$.

Theorem B.4. Let $\mathbf{x}=a_{0} \otimes \mathbf{h} \otimes \mathbf{a} \otimes 1_{E}$, with $\mathbf{a}=a_{1} \otimes \cdots \otimes a_{r} \in \bar{A}^{\otimes^{r}}$ and $\mathbf{h}=h_{0} \otimes \cdots \otimes h_{s} \in H \otimes \bar{H}^{\otimes^{s}}$. For $2 \leq l \leq s$, we have

$$
d_{r s}^{l}(\mathbf{x})=(-1)^{l(r+s)} a_{0} \otimes \mathbf{h}_{0, s-l} \otimes F_{r}^{(l)}\left(\mathbf{h}_{s-l+1, s}^{(1)} \otimes \mathbf{a}\right) \otimes 1 \# \mathfrak{h}_{s-l+1, s}^{(2)},
$$

where $F_{r}^{(l)}\left(\mathbf{h}_{s-l+1, s}^{(1)} \otimes \mathbf{a}\right)=F_{0}^{(l)}\left(\mathbf{h}_{s-l+1, s}\right)$ if $r=0$.

Proof. Let us compute $d_{r s}^{l+1}$ for $l \geq 1$. First we suppose the formula is valid for $d_{r s}^{j}$ with $j \leq l$ and we see that it is valid for $d_{0 s}^{l+1}$. To abbreviate we write $\zeta_{i}=$ $i s+(l-i+1)(s-1)+1$. Using the inductive hypothesis and the fact that $\sigma^{0} d^{l}\left(a_{0} \otimes\right.$ $\left.\mathbf{h}_{0 s} \otimes 1 \# 1\right)=0$, we obtain:

$$
\begin{aligned}
& d^{l+1}\left(1 \otimes \mathbf{h} \otimes 1_{E}\right)=-\sum_{i=1}^{l} \sigma^{0} d^{l+1-i} d^{i}\left(1 \otimes \mathbf{h} \otimes 1_{E}\right) \\
& =\sum_{i=1}^{l}(-1)^{i s+1} \sigma^{0} d^{l+1-i}\left(1 \otimes \mathbf{h}_{0, s-i} \otimes F^{(i)}\left(\mathbf{h}_{s-i+1, s}^{(1)}\right) \otimes 1 \# \mathfrak{h}_{s-i+1, s}^{(2)}\right) \\
& =\sum_{i=1}^{l} \sigma^{0}\left((-1)^{\zeta_{i}} \otimes \mathbf{h}_{0, s-l-1} \otimes F^{(l+1-i)}\left(\begin{array}{c}
F^{(i)}\left(\mathbf{h}_{s-i+1, s}^{(1)}\right) \\
\mathbf{h}_{s-l, s-i}^{(1)}
\end{array}\right) \otimes f\left(\mathfrak{h}_{s-l, s-i}^{(2)}, \mathfrak{h}_{s-i+1, s}^{(2)}\right) \# \mathfrak{h}_{s-l, s}^{(3)}\right) \\
& =(-1)^{(l+1) s} 1 \otimes \mathbf{h}_{0, s-l-1} \otimes F^{(l+1)}\left(\mathbf{h}_{s-l, s}^{(1)}\right) \otimes 1 \# \mathfrak{h}_{s-l, s}^{(2)},
\end{aligned}
$$

where the last equality follows from the definition of $\sigma^{0}$ and Lemma B.3. Now, we suppose the result is valid for $d_{r^{\prime} s}^{l+1}$ with $r^{\prime}<r$ and we show that it is valid for $d_{r s}^{l+1}$. To abbreviate we write $\zeta_{i}=i(r+s)+(l-i+1)(r+s-1)+1$.

$$
\begin{aligned}
d^{l+1}\left(1 \otimes \mathbf{h} \otimes \mathbf{a} \otimes 1_{E}\right)=-\sum_{i=0}^{l} \sigma^{0} d^{l+1-i} d^{i}\left(1 \otimes \mathbf{h} \otimes \mathbf{a} \otimes 1_{E}\right) \\
=(-1)^{r+1} \sigma_{0} d^{l+1}(1 \otimes \mathbf{h} \otimes \mathbf{a} \otimes 1)-(-1)^{r+s} \sigma_{0} d^{l}\left(1 \otimes \mathbf{h}_{0, s-1} \otimes \mathbf{a}_{s}^{h_{s}^{(1)}} \otimes 1 \# h_{s}^{(2)}\right) \\
-\sum_{i=2}^{l} \sigma^{0} d^{l+1-i}\left((-1)^{i(r+s)} \otimes \mathbf{h}_{0, s-i} \otimes F^{(i)}\left(\begin{array}{c}
\mathbf{a} \\
\mathbf{h}_{s-i+1, s}^{(1)}
\end{array}\right) \otimes 1 \# \mathbf{h}_{s-i+1, s}^{(2)}\right) \\
=(-1)^{r+1} \sigma_{0} d^{l+1}(1 \otimes \mathbf{h} \otimes \mathbf{a} \otimes 1) \\
-\sum_{i=1}^{l} \sigma^{0} d^{l+1-i}\left((-1)^{i(r+s)} \otimes \mathbf{h}_{0, s-i} \otimes F^{(i)}\left(\begin{array}{c}
\mathbf{h}_{s-i+1, s}^{(1)} \\
\mathbf{a}
\end{array}\right) \otimes 1 \# \mathbf{h}_{s-i+1, s}^{(2)}\right) \\
=\sigma^{0}\left((-1)^{(l+1)(r+s-1)+r+1} \otimes \mathbf{h}_{0, s-l-1} \otimes F^{(l+1)}\left(\begin{array}{c}
\mathbf{a}_{1, r-1} \\
\mathbf{h}_{s-l, s}^{(1)}
\end{array}\right) \otimes a_{r}^{\mathfrak{h}_{s-l, s}^{(2)}} \# \mathfrak{h}_{s-l, s}^{(3)}\right. \\
+ \\
\sum_{i=1}^{l}(-1)^{\zeta_{i}} \otimes \mathbf{h}_{0, s-l-1} \otimes F^{(l+1-i)}\left(\begin{array}{c}
F^{(i)} \mathbf{h}_{s-i+1, s}^{(1)} \\
\mathbf{h}_{s-l, s-i}^{(1)}
\end{array}\right) \otimes f\left(\mathbf{h}_{s-l, s-i}^{(2)}, \mathbf{h}_{s-i+1, s}^{(2)} \# \mathbf{h}_{s-l, s}^{(3)}\right) \\
=(-1)^{(l+1)(r+s)} \otimes \mathbf{h}_{0, s-l-1} \otimes F^{(l+1)}\left(\begin{array}{c}
\mathbf{a} \\
\mathbf{h}_{s-l, s}^{(1)}
\end{array}\right) \otimes 1 \# \mathfrak{h}_{s-l, s}^{(2)},
\end{aligned}
$$


where the last equality follows from the definition of $\sigma^{0}$ and Lemma B.3.

Remark B.5. When $H$ is a group algebra $k[G]$ and the 2-cocycle $f$ takes its values in the center of $A$, then

$d_{r s}^{l}\left(a_{0} \otimes \mathbf{g}_{0 s} \otimes \mathbf{a}_{1 r} \otimes 1_{E}\right)=(-1)^{l(r+s)} a_{0} \otimes \mathbf{g}_{0, s-l} \otimes F_{0}^{(l)}\left(\mathbf{g}_{s-l+1, s}\right) * \mathbf{a}_{1 r} \otimes 1 \# \mathfrak{g}_{s-l+1, s}$,

where $*$ denotes the shuffle product:

$\mathbf{a}_{1 r} * \mathbf{b}_{1 l}=\sum_{0 \leq i_{1} \leq \cdots \leq i_{r} \leq l}(-1)^{i_{1}+\cdots+i_{l}} b_{1} \otimes \cdots \otimes b_{i_{1}} \otimes a_{1} \otimes b_{i_{1}+1} \otimes \cdots \otimes b_{i_{r}} \otimes a_{r} \otimes b_{i_{r}+1} \otimes \cdots$

\section{REFERENCES}

[B] F. R. Beyl, The spectral sequence of a group extension, Bull. Sc. Math. 105(2) (1981), 417-434.

[B-C-M] R. J. Blattner, M. Cohen and S. Montgomery, Crossed products and inner actions of Hopf algebras, Trans. Amer. Math. Soc. 298 (1986), 671-711.

[Br] T. Brzeziński, Crossed products by a coalgebra, Comm. in Alg. 25 (1997), 3551-3575.

[D-T] Y. Doi and M. Takeuchi, Cleft comodule algebras by a bialgebra, Comm. in Alg. 14 (1986), 801-817.

[E] L. Evens, the spectral sequence of a finite group stops, Trans. Amer. Math. Soc. 212 (1975), 269-277.

[G-S] M. Gerstenhaber and S. D. Schack, Relative Hochschild cohomology, rigid algebras and the Bockstein, Journal of Pure and Applied Algebra 43 (1986), 53-74.

[G] A. Guichardet, Suites spectrales à la Hochschild-Serre pour les produits croisés d'algébres et de groupes, preprint.

[H-S] G. Hochschild and J. P. Serre, Cohomology of group extensions, Trans. Amer. Math. Soc. 74 (1953), 110-134.

[L] M. Lorenz, On the homology of graded algebras, Communications in Algebra 20(2) (1992), 489-507.

[Q] D. Quillen, Cyclic cohomology and algebra extensions, K-theory 3 (1989), 205-246.

[S] D. Stefan, Hochschild cohomology of Hopf Galois extensions, Journal of Pure and Applied Algebra 103 (1995), 221-233. 


\section{DeCOMPOSITIONS}

5.1. A decomposition of $\left(\widehat{X}_{*}, \widehat{d}_{*}\right)$. Let $[H, H]$ be the $k$-submodule of $H$ spanned by the set of all elements $a b-b a(a, b \in H)$. It is easy to see that $[H, H]$ is a coideal in $H$. Let $\breve{H}$ be the quotient coalgebra $H /[H, H]$. Given $h \in H$, we let $[h]$ denote the class of $h$ in $\breve{H}$. Given a subcoalgebra $C$ of $\breve{H}$ and a right $\breve{H}$-comodule $(N, \rho)$, we put $N^{C}=\{n \in N: \rho(n) \in N \otimes C\}$. It is well known that if $\breve{H}$ decomposes as a direct sum of subcoalgebras $C_{i}(i \in I)$, then $N=\bigoplus_{i \in I} N^{C_{i}}$.

Now, let us assume that $M$ is a Hopf bimodule. That is, $M$ is an E-bimodule and a right $H$-comodule, and the coaction $m \mapsto m^{(0)} \otimes m^{(1)}$ verifies:

$((a \# h) m(b \# l))^{(0)} \otimes((a \# h) m(b \# l))^{(1)}=\left(a \# h^{(1)}\right) m^{(0)}\left(b \# l^{(1)}\right) \otimes\left(a \# h^{(2)}\right) m^{(1)}\left(b \# l^{(2)}\right)$.

For each $n \geq 0, \widehat{X}_{n}$ is an $\breve{H}$-comodule via

$$
\begin{aligned}
& \rho_{n}\left(m \otimes_{A}\left(1 \# h_{1}\right) \otimes_{A} \cdots\left(1 \# h_{s}\right) \otimes \mathbf{a}_{1 r}\right) \\
& =m^{(0)} \otimes_{A}\left(1 \# h_{1}^{(1)}\right) \otimes_{A} \cdots\left(1 \# h_{s}^{(1)}\right) \otimes \mathbf{a}_{1 r} \otimes\left[m^{(1)} h_{1}^{(2)} \ldots h_{s}^{(2)}\right]
\end{aligned}
$$

where $r+s=n$. Moreover, the map $\rho_{*}: \widehat{X}_{*} \rightarrow \widehat{X}_{*} \otimes \breve{H}$ is a morphism of complexes. This fact implies that if $C$ is a subcoalgebra of $\breve{H}$, then $\widehat{d}_{n}\left(\widehat{X}_{n}^{C}\right) \subseteq \widehat{X}_{n-1}^{C}$. We consider the subcomplex $\left(\widehat{X}_{*}^{C}, \widehat{d}^{C}\right)$ of $\left(\widehat{X}_{*}, \widehat{d}_{*}\right)$, with modules $\widehat{X}_{n}^{C}$, and we let $\mathrm{H}_{*}^{C}(E, M)$ denote its homology. The filtration of $\left(\widehat{X}_{*}, \widehat{d}_{*}\right)$ introduced above Corollary 2.2 induces a filtration $F^{0}\left(\widehat{X}_{*}^{C}\right) \subseteq F^{1}\left(\widehat{X}_{*}^{C}\right) \subseteq \ldots$ on $\left(\widehat{X}_{*}^{C}, \widehat{d}_{*}^{C}\right)$. It is clear that $F^{i}\left(\widehat{X}_{n}^{C}\right)=\bigoplus_{0 \leq s \leq i} \widehat{X}_{n-s, s}^{C}$. Hence, we have a convergent spectral sequence

$$
E_{r s}^{1}=\mathrm{H}_{r}\left(A,\left(M \otimes_{A}(E / A)^{\left(\otimes_{A}\right)^{s}}\right)^{C}\right) \Rightarrow \mathrm{H}_{r+s}^{C}(E, M) .
$$

By the previous discussion, if $\breve{H}$ decomposes as a direct sum of subcoalgebras $C_{i}(i \in$ $I)$, then $\left(\widehat{X}_{*}, \widehat{d}_{*}\right)=\bigoplus_{i \in I}\left(\widehat{X}_{*}^{C_{i}}, \widehat{d}_{*}^{C_{i}}\right)$. Consequently $\mathrm{H}_{*}(E, M)=\bigoplus_{i \in I} \mathrm{H}_{*}^{C_{i}}(E, M)$ and the spectral sequence of Corollary 2.2 decomposes as direct sum of the above mentioned spectral sequences. Finally, the canonical normalized Hochschild complex $\left(M \otimes \bar{E}^{\otimes^{*}}, b_{*}\right)$ is a differential $\breve{H}$-comodule via

$\rho_{n}\left(m \otimes a_{1} \# h_{1} \otimes \cdots \otimes a_{n} \# h_{n}\right)=m^{(0)} \otimes a_{1} \# h_{1}^{(0)} \otimes \cdots \otimes a_{n} \# h_{n}^{(0)} \otimes\left[m^{(1)} h_{1}^{(1)} \cdots h_{n}^{(0)}\right]$

and the morphism from $\left(\widehat{X}_{*}, \widehat{d}_{*}\right)$ to $\left(M \otimes \bar{E}^{\otimes^{*}}, b_{*}\right)$, induced by the map $\phi_{*}$ of Section 1.2 , is an $\breve{H}$-comodule morphism. Hence, $\mathrm{H}_{*}^{C}(E, M)=\mathrm{H}_{*}\left(\left(\left(M \otimes \bar{E}^{\otimes^{*}}\right)^{C}, b_{*}\right)\right)$, for each subcoalgebra $C$ of $\breve{H}$. Note that the filtration of the normalized Hochschild complex given below Corollary 2.2 induces a filtration of $\left(\left(M \otimes \bar{E}^{\otimes^{*}}\right)^{C}, b_{*}\right)$. The spectral sequence of this filtration is isomorphic to the spectral sequence (1).

5.2. A decomposition of $\left(\bar{X}_{*}, \bar{d}_{*}\right)$. Now, suppose the cocycle $f$ is invertible. A direct computation shows that the $\breve{H}$-coaction of $\left(\bar{X}_{*}, \bar{d}_{*}\right)$, obtained transporting the one of $\left(\widehat{X}_{*}, \widehat{d}_{*}\right)$ through $\theta_{*}:\left(\widehat{X}_{*}, \widehat{d}_{*}\right) \rightarrow\left(\bar{X}_{*}, \bar{d}_{*}\right)$, is given by

(2) $m \otimes \mathbf{a} \otimes \mathbf{h} \mapsto m^{(0)} \otimes \mathbf{a} \otimes h_{1}^{(2)} \otimes \cdots \otimes h_{s}^{(2)} \otimes m^{(1)} S\left(h_{s}^{(1)}\right) \cdots S\left(h_{1}^{(1)}\right) h_{1}^{(3)} \cdots h_{s}^{(3)}$, 
where $\mathbf{a} \in \bar{A}^{\otimes^{r}}$ and $\mathbf{h}=h_{1} \otimes \cdots \otimes h_{s} \in \bar{H}^{\otimes^{s}}$. For each subcoalgebra $C$ of $\breve{H}$, we consider the subcomplex $\left(\bar{X}_{*}^{C}, \bar{d}_{*}^{C}\right)$ of $\left(\bar{X}_{*}, \bar{d}_{*}\right)$ with modules $\bar{X}_{n}^{C}$. It is clear that $\theta_{*}$ induces an isomorphism $\theta_{*}^{C}:\left(\widehat{X}_{*}^{C}, \widehat{d}_{*}^{C}\right) \rightarrow\left(\bar{X}_{*}^{C}, \bar{d}_{*}^{C}\right)$. Let $F^{0}\left(\bar{X}_{*}^{C}\right) \subseteq F^{1}\left(\bar{X}_{*}^{C}\right) \subseteq \ldots$ be the filtration of $\left(\bar{X}_{*}^{C}, \bar{d}_{*}^{C}\right)$ obtained transporting the one of $\left(\widehat{X}_{*}^{C}, \widehat{d}_{*}^{C}\right)$, through $\theta_{*}^{C}$. It is clear that $F^{i}\left(\bar{X}_{n}^{C}\right)=\bigoplus_{0 \leq s \leq i} \bar{X}_{n-s, s}^{C}$. Moreover, from (2) it follows that if $\breve{H}$ is cocommutative, then $\bar{X}_{r s}^{C}=M^{C} \otimes \bar{A}^{\otimes^{r}} \otimes \bar{H}^{\otimes^{s}}$. Hence, when $\breve{H}$ is cocommutative and $H$ is a flat $k$-module, the spectral sequence of (1) verifies $E_{r s}^{1}=\mathrm{H}_{r}\left(A, M^{C}\right) \otimes \bar{H}^{\otimes^{s}}$ and $E_{r s}^{2}=\mathrm{H}_{r}\left(H, \mathrm{H}_{s}\left(A, M^{C}\right)\right)$, where $\mathrm{H}_{r}\left(A, M^{C}\right)$ is a left $H$-module via the action introduced in Proposition 3.1.2.

5.3. An application to group crossed products. Let $G$ be a finite group, $E=A \#_{f} G$ a crossed product and $M=\bigoplus_{g \in G} M_{g}$ a $G$-graded $E$-bimodule. Let $\langle G\rangle$ be the set of conjugation classes of $G$. For each $g \in G$ we let $\langle g\rangle$ denote the conjugation class of $g$ and we write $M_{[g]}=\bigoplus_{h \in\langle g\rangle} M_{h}$. The complex $\left(\bar{X}^{*}, \bar{d}^{*}\right)$ decomposes as a direct sum of chain complexes $\left(\bar{X}^{*}, \bar{d}^{*}\right)=\bigoplus_{\langle g\rangle \in\langle G\rangle}\left(\bar{X}^{*}, \bar{d}^{*}\right)^{\langle g\rangle}$, where

$$
\left(\bar{X}^{*}, \bar{d}^{*}\right)^{\langle g\rangle}=\bigoplus_{r+s=n} \operatorname{Hom}_{k}\left(\bar{A}^{\otimes^{r}} \otimes \overline{k[G]}^{\otimes^{s}}, M_{[g]}\right)
$$

Hence $\mathrm{H}^{n}(E, M)=\bigoplus_{\langle g\rangle \in\langle G\rangle} \mathrm{H}^{n}\left(\left(\bar{X}^{*}, \bar{d}^{*}\right)^{\langle g\rangle}\right)$. The filtration introduced in Corollary 3.2.3 induces a filtration in each one of the terms of the decomposition of $\left(\bar{X}^{*}, \bar{d}^{*}\right)$. Taking the spectral sequences of these filtrations we get converging spectral sequences

$$
E_{2}^{r s}=\mathrm{H}^{s}\left(G, \mathrm{H}^{r}\left(A, M_{[g]}\right)\right) \Rightarrow \mathrm{H}^{r+s}\left(\left(\bar{X}^{*}, \bar{d}^{*}\right)^{\langle g\rangle}\right) \quad\langle g\rangle \in\langle G\rangle .
$$

Now, it is immediate that the spectral sequence of Corollary 3.2.3 is the direct sum of these spectral sequences. Finally the canonical complex $\left(\operatorname{Hom}_{k}\left(\bar{E}^{\otimes^{*}}, M\right), b^{*}\right)$ decomposes as a direct sum $\left(\operatorname{Hom}_{k}\left(\bar{E}^{\otimes^{*}}, M\right), b^{*}\right)=\bigoplus_{\langle g\rangle \in\langle G\rangle}\left(\operatorname{Hom}_{k}\left(\bar{E}^{\otimes^{*}}, M\right)^{\langle g\rangle}, b^{*}\right)$, where

$$
\operatorname{Hom}_{k}\left(\bar{E}^{\otimes^{n}}, M\right)^{\langle g\rangle}=\bigoplus_{\substack{g_{1}, \ldots, g_{n+1} \in G \\ g_{n}^{-1} \cdots g_{1}^{-1} g_{n+1} \in\langle g\rangle}} \operatorname{Hom}_{k}\left(\overline{A \# g_{1}} \otimes \cdots \otimes \overline{A \# g_{n}}, M_{g_{n+1}}\right)
$$

We assert that $\mathrm{H}^{n}\left(\left(\bar{X}^{*}, \bar{d}^{*}\right)^{\langle g\rangle}\right)=\mathrm{H}^{n}\left(\left(\operatorname{Hom}_{k}\left(\bar{E}^{\otimes^{*}}, M\right)^{\langle g\rangle}, b^{*}\right)\right)$. In fact, this follows from the fact that the complex $\left(\widehat{X}^{*}, \widehat{d}^{*}\right)$ introduced in Section 2 decomposes as direct sums of chain complexes $\left(\widehat{X}^{*}, \widehat{d}^{*}\right)=\bigoplus_{\langle g\rangle \in\langle G\rangle}\left(\widehat{X}^{*}, \widehat{d}^{*}\right)^{\langle g\rangle}$, where

$$
\widehat{X}^{n}=\bigoplus_{r+s=n} \bigoplus_{\substack{g_{1}, \ldots, g_{s+1} \in G \\ g_{s}^{-1} \cdots g_{1}^{-1} g_{s+1} \in\langle g\rangle}} \operatorname{Hom}_{k}\left(\bar{A}^{\otimes^{r}} \otimes k g_{1} \otimes \cdots \otimes k g_{s}, M_{g_{s+1}}\right)
$$

and from the fact that the morphism $\theta^{*}:\left(\bar{X}^{*}, \bar{d}^{*}\right) \rightarrow\left(\widehat{X}^{*}, \widehat{d}^{*}\right)$ introduced in Theorem 3.2.1 and the morphism from $\left(\operatorname{Hom}_{k}\left(\bar{E}^{\otimes^{*}}, M\right), b^{*}\right)$ to $\left(\widehat{X}^{*}, \widehat{d}^{*}\right)$, induced by the map $\phi_{*}$ of Section 1.2, preserve the decompositions. 


\section{Miscelaneas}

2.1.4. Compatibility with the canonical decomposition. Let us assume that $k \supseteq \mathbb{Q}, H$ is cocommutative, $A$ is commutative, $M$ is symmetric as an $A$-bimodule and the cocycle $f$ takes its values in $k$. In [G-S1] was obtained a decomposition of the canonical Hochschild complex $\left(M \otimes \bar{A}^{\otimes^{*}}, b_{*}\right)$. It is easy to check that the maps $\widehat{d_{0}}$ and $\widehat{d}_{1}$ are compatible with this decomposition. Since $\widehat{d}_{l}=0$ for all $l \geq 2$, we obtain a decomposition of $\left(\widehat{X}_{*}, \widehat{d}_{*}\right)$, and then a decomposition of $\mathrm{H}_{*}(E, M)$.

2.2.3. Compatibility with the canonical decomposition. Assume that $k \supseteq$ $\mathbb{Q}, H$ is cocommutative, $A$ is commutative, $M$ is symmetric as an $A$-bimodule and the cocycle $f$ takes its values in $k$. Then, the Hochschild cohomology $\mathrm{H}^{*}(E, M)$ has a decomposition similar to the one obtained in 2.1.4 for the Hochschild homology.

3.1.7. An application to $\operatorname{Tor}_{*}^{E}$. Let $k$ be a field, $B$ an arbitrary $k$-algebra, $M$ a right $B$-module and $N$ a left $B$-module. It is well known that $\operatorname{Tor}_{*}^{B}(M, N) \simeq$ $\mathrm{H}_{*}(B, N \otimes M)$ (here $N \otimes M$ is an $B$-bimodule via $\left.a(n \otimes m) b=a n \otimes m b\right)$. This fact and Corollary 3.1.3 show that if $k$ is a field, $M$ is a right $E$-module and $N$ is a left $E$-module, then there is a convergent spectral sequence

$$
E_{r s}^{2}=\mathrm{H}_{r}\left(H, \operatorname{Tor}_{s}^{A}(M, N)\right) \Rightarrow \operatorname{Tor}_{r+s}^{E}(M, N) .
$$

3.2.6. An application to $\operatorname{Ext}_{E}^{*}$. Let $k$ be a field, $B$ an arbitrary $k$-algebra and $M$, $N$ two left $B$-modules. It is well known that $\operatorname{Ext}_{B}^{*}(M, N) \simeq \mathrm{H}^{*}\left(B, \operatorname{Hom}_{k}(M, N)\right)$ (here $\operatorname{Hom}_{k}(M, N)$ is an $B$-bimodule via $\left.(a \varphi b)(m)=a \varphi(b m)\right)$. This fact and Corollary 3.2.3 show that if $k$ is a field and $M$ and $N$ are left $E$-modules, then there is a convergent spectral sequence

$$
E_{2}^{r s}=\mathrm{H}^{r}\left(H, \operatorname{Ext}_{A}^{s}(M, N)\right) \Rightarrow \operatorname{Ext}_{E}^{r+s}(M, N)
$$

As a corollary we obtain that gl. $\operatorname{dim}(E) \leq$ gl. $\operatorname{dim}(A)+$ gl. $\operatorname{dim}(H)$, where gl. $\operatorname{dim}$ denotes the left global dimension. Note that this result implies Maschke's Theorem for crossed product, as it was established in [B-M].

Jorge Alberto Guccione, Departamento de Matemática, Facultad de Ciencias Exactas y Naturales, Pabellón 1 - Ciudad Universitaria, (1428) Buenos Aires, ArGENTINA.

E-mail address: vander@dm.uba.ar

Juan José Guccione, Departamento de Matemática, Facultad de Ciencias Exactas Y Naturales, Pabellón 1 - Ciudad Universitaria, (1428) Buenos Aires, Argentina.

E-mail address: jjgucci@dm.uba.ar 